\title{
The Xer activation factor of TLCФ expands the possibilities for Xer recombination
}

Solange Miele ${ }^{1}$, Justine Vergne ${ }^{1}$, Christophe Possoz ${ }^{1}$, Françoise Ochsenbein ${ }^{1}$ and FrançoisXavier Barre*1

${ }^{1}$ Institute for Integrative Biology of the Cell (I2BC), Université Paris-Saclay, CEA, CNRS, Université Paris Sud, 1 Avenue de la Terrasse, 91198 Gif-sur-Yvette, France

*Correspondence to: francois-xavier.barre@i2bc.paris-saclay.fr

Nb characters: 51169

Nb figures: 5 


\section{ABSTRACT}

Many mobile elements take advantage of the highly-conserved chromosome dimer resolution system of bacteria, Xer. They participate in the transmission of antibiotic resistance and pathogenicity determinants. In particular, the toxin-linked cryptic satellite phage (TLCФ) plays an essential role in the continuous emergence of new toxigenic clones of the Vibrio cholerae strain at the origin of the ongoing $7^{\text {th }}$ cholera pandemic. The Xer machinery is composed of two chromosomally-encoded tyrosine recombinases, XerC and XerD. They resolve chromosome dimers by adding a crossover between sister copies of a specific 28 base pair site of bacterial chromosomes, dif. The activity of XerD depends on a direct contact with a cell division protein, FtsK, which spatially and temporally constrains the process. TLC $\Phi$ encodes for a XerD-activation factor (XafT), which drives the integration of the phage into the dif site of the primary chromosome of $V$. cholerae independently of FtsK. However, XerD does not bind to the attachment site (attP) of TLC $\Phi$, which raised questions on the integration process. Here, we compared the integration efficiency of thousands of synthetic mini-TLCФ plasmids harbouring different attP sites and assessed their stability in vivo. In addition, we compared the efficiency with which XafT and the XerD activation domain of FtsK drive recombination reactions in vitro. Taken together, our results suggest that XafT promotes the formation of synaptic complexes between canonical Xer recombination sites and imperfect sites. 


\section{INTRODUCTION}

Repair by homologous recombination can lead to the formation of chromosome dimers when the chromosomes are circular, as is generally the case in bacteria and archaea. Chromosome dimers physically impede the segregation of genetic information. They are resolved by the addition of a crossover at a specific locus, dif, by a highly conserved chromosomally encoded tyrosine recombination (Xer) machinery (1).

Many mobile elements take advantage of the high conservation of the Xer machinery (1). Indeed, it was initially characterized as a multicopy plasmid dimer resolvase $(2,3)$. Diverse

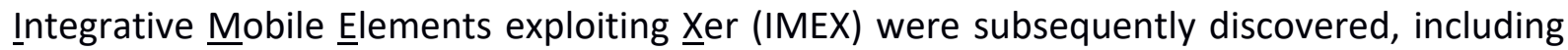
phages and genetic islands that harbour a dif-like attachment site (attP) and integrate into the dif site of one of the chromosomes of their host $(4,5)$. Plasmids and IMEX participate in the acquisition of antibiotic resistance and pathogenicity determinants. In particular, the principal virulence factor of Vibrio cholerae, cholera toxin, is encoded in the genome of a lysogenic phage (CTXФ), which exploits Xer for integration $(6,7)$. Several other IMEX contribute to the continuous emergence of new toxigenic clones of the $V$. cholerae strain at the origin of the ongoing $7^{\text {th }}$ cholera pandemic (8-11). Foremost among those is a toxin-linked cryptic satellite phage (TLCD) whose integration is thought to correct the dif site of the primary chromosome of non-toxigenic environmental $V$. cholerae strains, difA, into a site suitable for the integration of CTXФ, dif1 (Figure 1A, $(10,11)$ ).

In V. cholerae, as in most bacteria, the Xer machinery is composed of two closely related recombinases, XerC and XerD (Figure S1A). The dif sites are composed of two partiallypalindromic 11 base pair (bp) XerC and XerD binding arms separated by a short 6 bp central region (Figure 1B and S1B). XerC and XerD each catalyse the cleavage and transfer of a specific pair of DNA strands, which are referred to as the top and bottom strands, respectively (Figure 
1B). Chromosome dimer resolution follows the conventional recombination pathway of tyrosine recombinases, with the cleavage and transfer of one pair of strands leading to the formation of an obligatory Holliday junction (HJ) intermediate that is subsequently resolved into crossover by the exchange of a second pair of strands (Figure 1C). The first pair of strands is catalysed by XerD and the second by XerC (Figure 1C, $(12,13)$ ). The process is under the control of a DNA translocase anchored in the cell division septum, FtsK, which activates XerD by a direct contact with its C-terminal domain, FtsKy (Figure 1C, (12, 14-16)). Hence, Xer recombination is normally restricted to the time of cell division and to synaptic complexes located in the proximity of the cell division apparatus (17-20). However, many IMEX, including CTXФ, integrate via a non-conventional FtsK-independent recombination pathway: they exploit the low basal ability of XerC to catalyse the formation of HJs, which are subsequently resolved by replication (Figure $1 \mathrm{C},(8,9,21)$ ). Dimers of the ColE1 multicopy plasmid are resolved by a similar process (22). In contrast, TLCФ integration follows the same recombination pathway as chromosome dimer resolution (10). However, the process escapes the FtsK control (10) because ILCФ encodes for its own XerD activation factor, XafT (Figure 1C, (23)).

Eight base pairs of the XerD-arm of TLCФ attP deviate from the canonical XerD-arm of dif1 and difA (Figure 1B and S1B). It was proposed to prevent undesired FtsK-driven prophage excision, at least in part because FtsK can dismantle non-canonical synaptic complexes when it translocates on the genome of the integrated IMEX (Figure 1D, (24)). However, the XerD arm of TLCФ attP abolishes XerD binding, which questioned the possibility for integration (Figure 1D, (10, 11, 23)).

Here, we analysed the influence of the sequence of the XerD arm of TLCФ attP on FtsK- and XafT-driven recombination reactions by comparing the integration efficiency of thousands of 
bioRxiv preprint doi: https://doi.org/10.1101/2021.09.09.459576; this version posted September 9,2021 . The copyright holder for this preprint (which was not certified by peer review) is the author/funder, who has granted bioRxiv a license to display the preprint in perpetuity. It is made available under aCC-BY-NC-ND 4.0 International license.

synthetic mini-TLCФ plasmids harbouring attP sites with differing XerD-arms and assessing their stability in vivo. In addition, we analysed the efficiency with which XafT and FtsK $\gamma$ drive recombination reactions between two dif1 sites and between dif1 and TLC $\Phi$ attP. Taken together, our results suggest that XafT promotes the formation of synaptic complexes between dif sites and imperfect Xer recombination sites.
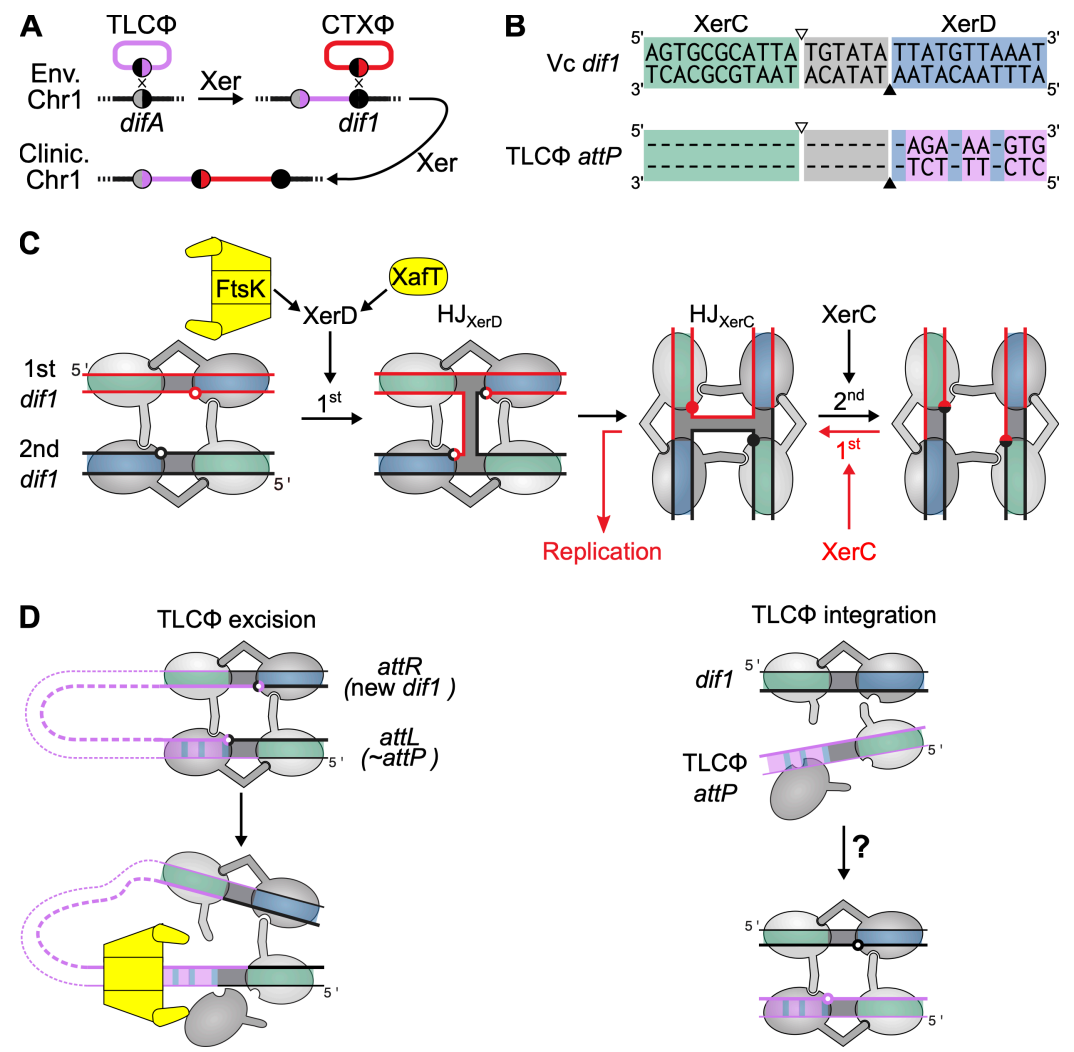

Figure 1. Xer recombination. (A) Toxigenic conversion of $V$. cholerae. Env. and Clinic. Chr1: primary chromosome of environmental and clinical $V$. cholerae. (B) TLCФ attP and $V$. cholerae dif1 sequence. Grey: central region; Green: XerC arm; Blue: XerD arm; Pink: TLCФ non-canonical bp; white and black triangles: XerC and XerD cleavage sites. (C) Xer recombination pathways. Black arrows: conventional recombination pathway; Red arrows: nonconventional asymmetric recombination pathway. The XerD and XerC cleavage points are depicted by empty and filled disks. (D) TLCФ excision/integration balance. Left: FtsK dismantles the excision complex when it translocates on the prophage DNA; Right: the non-canonical XerD-arm of TLC $\Phi$ attP abolishes binding of XerD. 

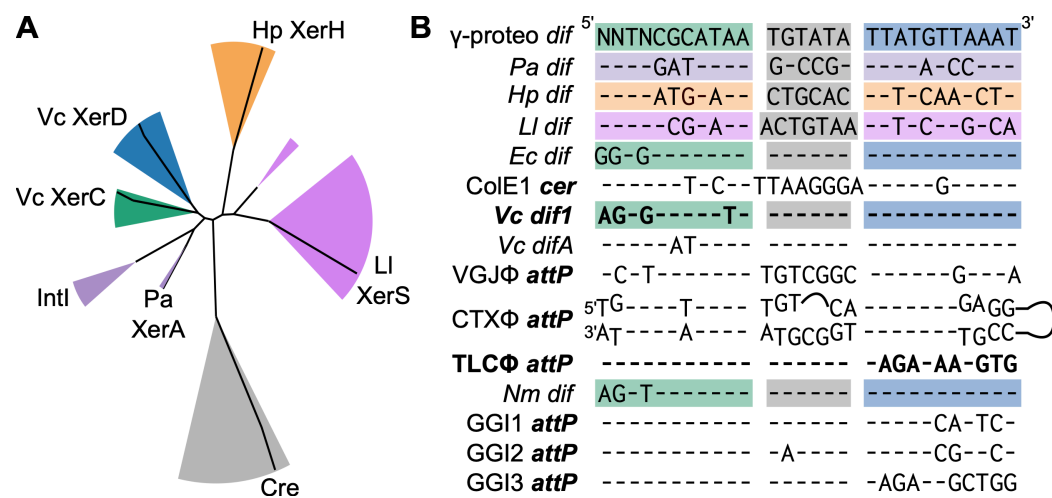

Supplementary Figure S1. Xer recombination. (A) Phylogeny of Xer recombinases. Grey, Green, Blue, Orange, Pink and Magenta sectors: Cre (outgroup), XerC, XerD, XerH, XerS, XerA and IntI families, respectively. Vc: $V$. cholerae; Hp: Helicobacter pylori; LI: Lactoccocus lactis; Pa: Pyrococcus abessi. (B) Sequence of the top strand of bacterial dif sites and of Xer recombination sites harboured by typical mobile genetic elements. The top strand is the site cleaved by XerC during recombination. Grey: central region; Green, Blue, Magenta, Orange and Pink: XerC-, XerD-, Pa XerA-, Hp XerH- and LI XerS-binding arms, respectively. $\gamma$-proteo: $\gamma$-proteobacteria dif consensus. ColE1 cer: core of the ColE1 plasmid dimer resolution site. Attachment site bases homologous to the host dif sequence are indicated by a hyphen. CTXФ attP is the stem of a folded hairpin, with 12 nt on the top strand of its central region and 7 on its bottom strand. Nm: Neisseria meningitidis.

\section{RESULTS}

\section{Methodology for parallel monitoring of the integration efficiency and stability}

We developed a methodology based on Next Generation Sequencing (NGS) to analyse the impact of the sequence of the XerD-arm of TLCФ attP on the integration efficiency and stability of the phage. In brief, we generated three pools of $65,536\left(4^{8}\right)$ degenerate attP sites using synthetic oligonucleotides carrying 8 degenerate bases at different positions of the XerD-arm of TLCФ attP as templates (Figure 2A). Two of the pools, referred to as $\mathrm{n}_{8} \mathrm{gtg}$ and $\operatorname{tagn}_{8}$ on the basis of their top strand, were designed to explore the influence of the eight innermost and outermost positions of the XerD-arm. Results were completed with a pool harbouring degenerate bases in both the inner and outer part of the XerD-arm, $n_{5} a_{2} n_{3} g$. The pools were cloned in place of the attP site of previously designed $\mathrm{XafT}^{+}$and $\mathrm{XafT}^{-}$conjugative suicide mini-TLCФ plasmids (23). NGS analysis showed that each resulting mini-TLCФ library contained over $99.5 \%$ of the XerD-arm sequence motifs covered by the pool from which it originated, with a median copy number in the order of 15 per million reads (Figure $2 \mathrm{~A}$ ). It 
further showed that the XafT ${ }^{+}$and XafT- mini-TLCФ libraries contained over $99.9 \%$ and $99.8 \%$ of the 190,528 XerD-arm sequence motifs covered by the three combined degenerate pools, respectively. The mini-TLCФ libraries were conjugated in two N16961 reporter strains harbouring an E. coli lacZ $\alpha$-dif1-lacZ $\beta$ gene fusion at the natural integration locus of TLCФ or at the lacZ locus (Figure S2, (10)). As the central region and XerC-binding arm of TLCФ attP and dif1 are identical, the attR and attL site resulting from the integration of the mini-TLC $\Phi$ plasmids are identical to their attP site and dif1, respectively. Thus, the integration and excision frequencies of the mini-TLCФ plasmids reflect the efficiency of intermolecular and intramolecular recombination reactions between the same two sites, respectively. The global frequency of dif1-integration events of each mini-TLC $\Phi$ library, $f$, was measured with a blue/white screen (Figure 2B). The relative proportion of each attP sequence motif in the genomic DNA of the recipient cells, $P_{\text {int }}$, was determined by NGS using a primer binding in $E$. coli lacZ and a primer binding in TLCФ (Figure 2B). The integration frequency of the corresponding mini-TLCФ plasmids was then estimated as $f_{\text {int }}=f \times P_{\text {int }}$. The reporter strains were further engineered to place production of XerC and XerD under the control of the arabinose promoter in order to prevent Xer-mediated excision after integration (Figure 2B, (25)). The stability of the integrated plasmids was estimated by analysing the proportion of each attP sequence motif in the cell population after growth in the presence of arabinose, $P_{\text {exc }}$ (Figure 2B). 

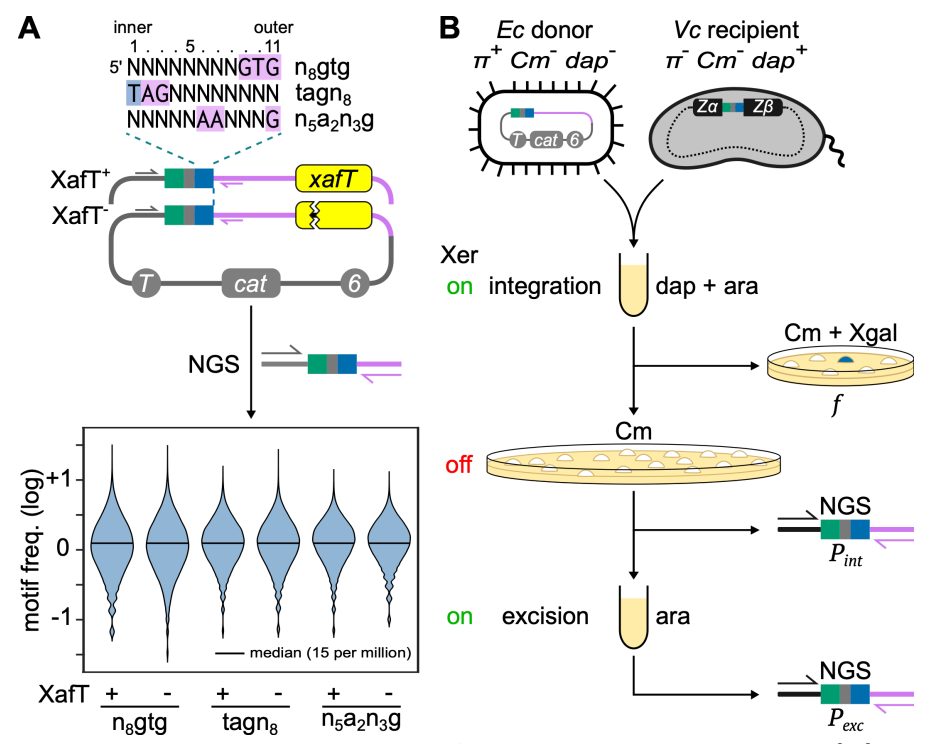

Figure 2. Parallel monitoring of integration and stability. (A) Mini-TLCФ plasmid libraries. Top panel: top strand of the degenerate attP motifs. Legend as in Figure 1. Middle panel: scheme of XafT ${ }^{+}$and XafT'mini-TLCФ plasmids. Pink: TLCФ DNA; Grey: plasmid DNA. Yellow rectangle: xafT gene; sawed lines: stop mutation; T: RP4 transfer origin; 6: pir-dependent replication origin; cat: chloramphenicol resistance gene. Plasmid-specific P5 and TLCФspecific P7 adaptor primers used for next generation sequencing (NGS) are indicated by grey and pink arrows, respectively. Bottom panel: distribution frequency of the motifs. (B) Integration and excision assays. On and off: growth in the presence or absence of arabinose. $\pi$ : R6K replication initiator; dap: diaminopimelic acid; $\mathrm{Cm}$ : chloramphenicol; Xgal: X-gal; Z $\alpha$ and Z $\beta$ : E. coli lacZ gene. The lacZ $\alpha$-specific P5 adaptor primer is indicated by a black arrow.

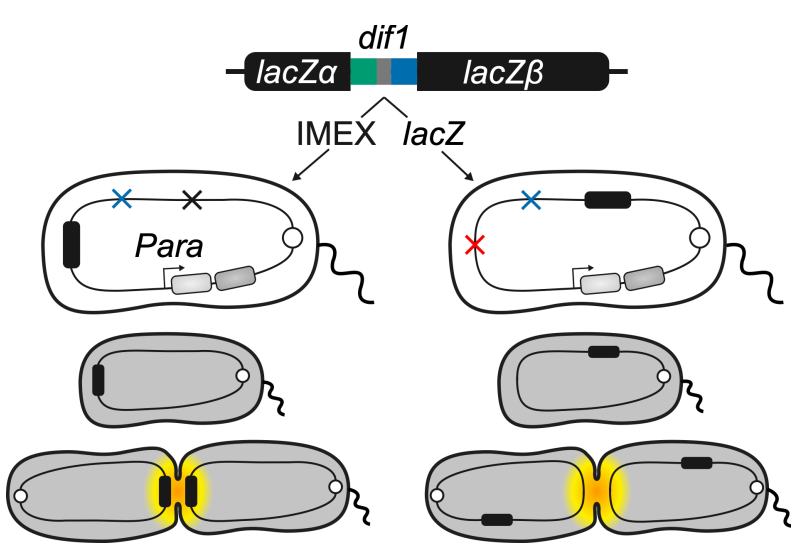

Supplementary Figure S2. Parallel monitoring of integration and stability. Scheme of the V. cholerae reporter strains. White circle: chromosome 1 replication origin; red, blue and black crosses: IMEX, xerD and lacZ deletions, respectively; black rectangle: E. coli lacZ $\alpha$-dif1-lacZ $\beta$ gene; light and dark grey rectangles: synthetic xerC and xerD operon under the control of the arabinose promoter (Para). Grey and yellow shadings depict the subcellular region and timing of activity of FtsK during the cell cycle.

\section{FtsK-driven integration is restricted to the sites that most resemble dif1}

To explore the influence of the sequence of the XerD-arm of TLCD attP on the efficiency of FtsK-driven integration events, we conjugated the XafT- mini-TLCФ libraries in the $V$. cholerae recipient strain harbouring dif1 at its natural locus (Figure 3, FtsK panel). The global integration 
frequency of the XafT- $n_{8}$ gtg, tagn $n_{8}$ and $n_{5} a_{2} n_{2} g$ mini-TLCФ libraries was 1000 -fold lower than that of a XafT- mini-TLCФ plasmid harbouring dif1 (Figure 3A, FtsK panel). NGS further revealed that $2.5 \%$ of the different possible attP sites comprised in the three XafT- libraries could be integrated (Figure 3A, FtsK panel).

To visualise the sequence bias of the XerD arm of the attP sites of the integrated plasmids, we attributed unique $x$ and $y$ coordinates to each XerD arm motif based on its sequence (Figure 3B, bottom scheme). We then drew two-dimensional maps (2D-maps) by colouring each $\mathrm{x}$ and y position based on the integration frequency of the corresponding attP site, from dark blue to bright yellow. The position of non-recovered attP sites were coloured in black. The density of the coloured positions highlighted the limited number of XerD-arm sequences that could be integrated by FtsK (Figure 3B and S3A, FtsK panels). A single small yellow tile was visible on the $n_{8}$ gtg and $n_{5} a_{2} n_{2} g$ 2D-maps, at the coordinates of the 64 TTATGNNNNGTG and 64 TTATGAANNNG top strand sequences, respectively. A larger blue tile was visible on the tagn 2D-map, which corresponded to the 1024 TAGTGTNNNNN top strand sequence coordinates. The lower frequency of integration and higher number of motifs recovered with the tagn 8 library showed that inner XerD positions played a more important role on FtsK-driven integration than outer positions.

Results were further analysed using butterfly plots, in which y-axis positions indicate the integration frequency of the sites and $\mathrm{x}$-axis positions indicate their number of differences from TLCФ attP, with positive and negative values attributed to sites that are closer from dif1 than TLCФ attP (Figure 3C and S3B, FtsK panels). While only a third of the attP sites of the $\mathrm{n}_{8} \mathrm{gtg}, \mathrm{n}_{5} \mathrm{a}_{2} \mathrm{n}_{2} \mathrm{~g}$ and tagn $\mathrm{n}_{8}$ libraries were closer from dif1 than TLCФ attP (21 070/ 65 536), over $98 \%$ of the sites whose integration could be driven by FtsK belonged to + wing of the plots (Figure 3C and S3B, FtsK panel). In addition, those sites accounted for over $99.6 \%$ of the 
integration events (Figure $3 \mathrm{C}$ and S3B, FtsK panel). We assigned a red, orange, cyan and blue colour code to the attP sites that integrated at dif1 by an FtsK-driven reaction at frequencies higher than $10^{-6}, 10^{-7}, 510^{-8}$ or lower than $510^{-8}$, respectively. The red sites comprised 53 $\mathrm{n}_{8} \mathrm{gtg}, 61 \mathrm{n}_{5} \mathrm{a}_{2} \mathrm{n}_{2} \mathrm{~g}$ and 61 tagn $_{8}$ sequences, which represented a total of 174 different sequences. We calculated the frequency of the four DNA bases at each position of the top strand of the XerD arm of the red sites by pooling together the sequences coming from libraries in which the position was degenerated. In all but the $11^{\text {th }}$ outermost position of the XerD arm, the dif1 base was the most frequent (Figure 3D, FtsK panel).

Together, those results show that FtsK-driven integration is restricted to the sites that most resemble dif1. Nevertheless, the NGS-based integration assay was sensitive enough to monitor the integration frequency of the XafT- mini-TLCФ plasmid harbouring TLCФ attP, in contrast to the classical blue/white assay (Figure 3, FtsK panels). The integration frequency of TLCФ attP was over a 1000 -fold lower than the mean integration frequency of the $\mathrm{n}_{8} \mathrm{gtg}$ and $\mathrm{n}_{5} \mathrm{a}_{2} \mathrm{n}_{2} \mathrm{~g}$ red attP sites, and over a 100 -fold lower than the mean integration frequency of the red tagn ${ }_{8}$ attP sites (Figure $3 \mathrm{C}$ and S3C, FtsK panel).

\section{XafT drives the integration of any attP site harbouring the same $5^{\text {th }}$ XerD-arm bp than dif1}

Previous works showed that the choreography of chromosome segregation restricts the activity of Ftsk to the terminus region of the two $V$. cholerae chromosomes, suggesting that FtsK could not drive integration at the lacZ locus (Figure S2, (25-28)). Correspondingly, no dif1specific integration events were observed at the lacZ locus with XafT- mini-TLCФ libraries. Thus, we could explore the influence of the sequence of the XerD-arm of TLC $\Phi$ attP on the efficiency of XafT-driven integration events by conjugating the $\mathrm{XafT}^{+}$mini-TLCФ libraries in the V. cholerae recipient strain harbouring dif1 at the lacZ locus (Figure 3, XafT panels). 
The integration of the XafT ${ }^{+}$dif1 and XafT ${ }^{+}$TLCФ attP plasmids at the lacZ locus was as efficient as the integration of the XafT- dif1 plasmid at the dif1 locus (Figure 3A, XafT panel). In addition, the global integration frequency of the XafT ${ }^{+} n_{8} g t g$, tagn $n_{8}$ and $n_{5} a_{2} n_{2} g$ mini-TLC $\Phi$ plasmid libraries was only 10-fold lower than that of the XafT ${ }^{+}$dif1 plasmid (Figure 3A, XafT panel). NGS analysis further revealed that over $25 \%$ of the different possible attP sites comprised in each of the three $\mathrm{XafT}^{+}$mini-TLCФ libraries could be integrated at the lacZ locus (Figure 3A, XafT panel).

The $n_{8} g t g$ and $n_{5} a_{2} n_{2} g$ 2D-maps presented a very striking chequered pattern, with the black stripes corresponding to members of the library that lacked a $G$ at the $5^{\text {th }}$ position of the top strand of the XerD-arm (Figure 3B and S3A, XafT panel). Likewise, the checker pattern of the $\operatorname{tagn}_{8} 2 \mathrm{D}$ maps reflected the importance of the nature of the residue at this position. Thus, XafT promoted the integration of $1 / 4$ of the attP library, corresponding to those that carried the same residues as dif 1 at the $5^{\text {th }}$ position of the XerD arm.

The colour of the $\mathrm{XafT}^{+}$2D maps patterns ranged from yellow to green, showing that XafTdriven integration was more efficent than XafT-driven integration (Figure 3B and S3A, XafT panel). In particular, the integration frequency of TLC $\Phi$ attP jumped from about $10^{-9}$ to about $10^{-6}$ (Figure $3 \mathrm{C}$ and S3B, XafT panels). In addition, differences in the frequency of attP sites from the red and orange categories were alleviated. In particular, the mean frequency of the red $n_{8} g t g$ and $n_{5} a_{2} n_{2} g$ attP sites was now only 30 -fold higher than that of TLCФ attP (Figure 3C and S3B, XafT panel). Furthermore, the integration frequency of many of the newly recovered sites, shown in grey, was higher than $10^{-6}$. Those sites were not limited to the positive wing of the butterfly plots: XafT drove the integration of about $30 \%$ of the $n_{8} g t g$ and $n_{5} a_{2} n_{2} g$ miniTLCФ and about $20 \%$ of the tagn 8 mini-TLCФ that belonged to the negative group (Figure $3 C$ 
and S3B, XafT). As a result, over 6000 different attP sites were found integrated at a frequency higher than $10^{-6}$, with a frequency logo that lost similarity to dif1 (Figure 3D, XafT panel).

\section{FtsK does not perturb XafT-mediated integration at the dif1 locus}

XafT ensured the efficient integration of many attP sites other than TLC $\Phi$ attP (Figure $3 C$ and S3, XafT panels). Therefore, we wondered whether TLCФ attP had been selected to avoid any perturbation of FtsK on the integration of the phage at the natural dif1 locus. To explore this possibility, we analysed the influence of the sequence of the XerD-arm of TLC $\Phi$ attP on the efficiency of integration of $\mathrm{XafT}^{+}$mini-TLCФ libraries that were conjugated in the $V$. cholerae recipient strain harbouring dif1 at its natural locus (Figure 3 and S3, XafT \& FtsK panels). The 2D maps and butterfly plots were similar to those obtained when the libraries were conjugated in the $V$. cholerae recipient strain harbouring dif1 at the lacZ locus (Figure 3 and S3, XafT \& FtsK panels). The only notable difference was a slightly higher global integration frequency (Figure 3A and S3, XafT \& FtsK panel). This is most probaly explained by the growth advantage of the reporter strain carrying dif1 at its natural locus over the reporter strain carrying dif1 at it's the lacz locus, since the later cannot resolve dimers of its primary chromosome (13). As a result, over 20000 different attP sites integrated at a frequency higher than $10^{-6}$, which decreased the similitude between the sequence logo and dif1 (Figure 3D, XafT \& FtsK panel). 
bioRxiv preprint doi: https://doi.org/10.1101/2021.09.09.459576; this version posted September 9,2021 . The copyright holder for this preprint (which was not certified by peer review) is the author/funder, who has granted bioRxiv a license to display the preprint in perpetuity. It is made available under aCC-BY-NC-ND 4.0 International license.

A

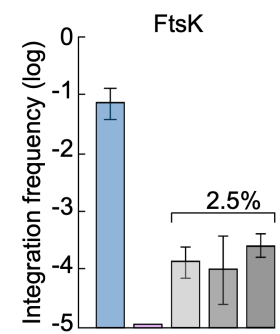

$\mathbf{B}$
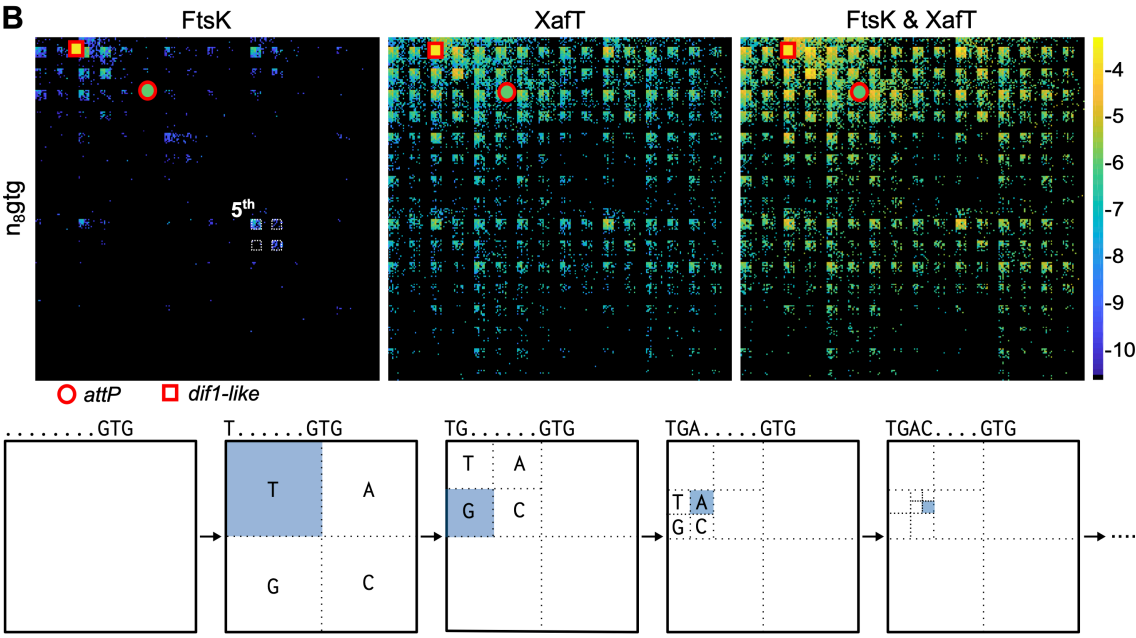

C
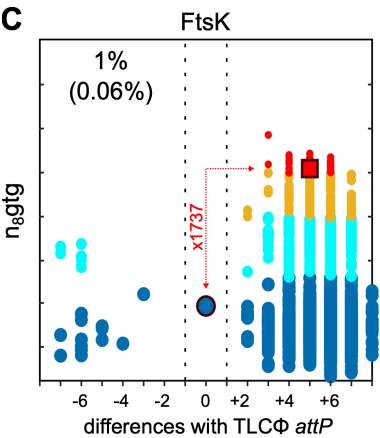

D

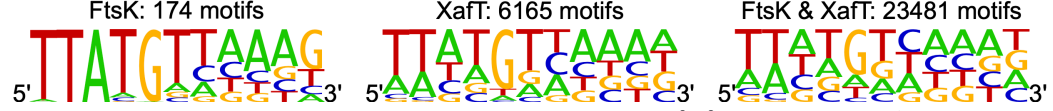

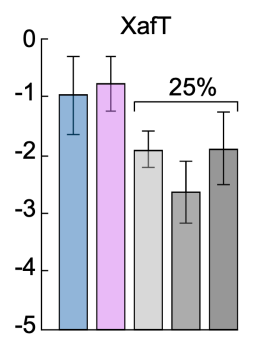

XafT

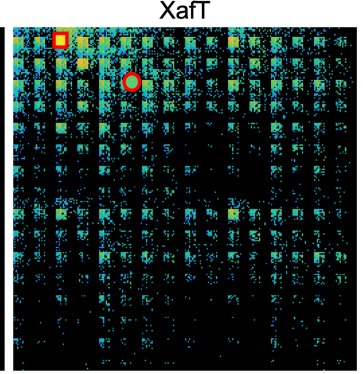

FtsK \& XafT
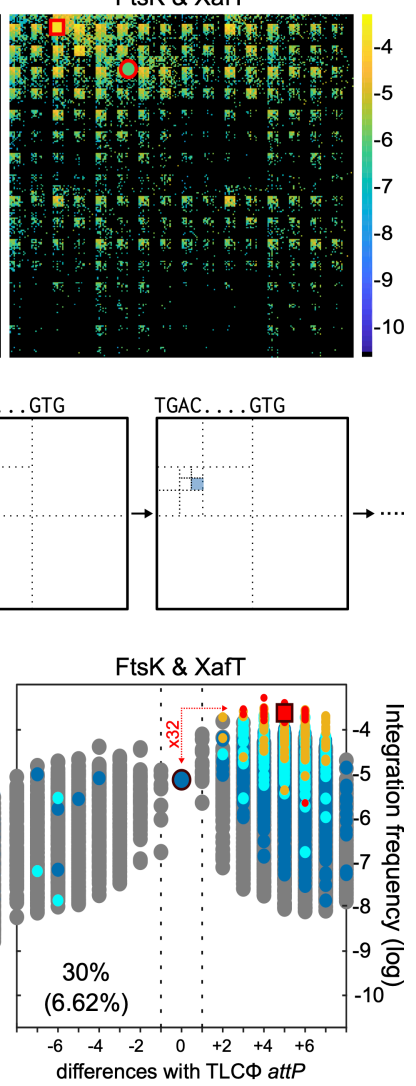

Figure 3. Suicide mini-TLCФ plasmid integration. (A) Integration frequencies of mini-TLCФ plasmids harbouring dif1 or TLCФ attP, and global integration frequencies $(f)$ of $n_{8} g t g, n_{5} a_{2} n_{2} g$ and tagn $n_{8}$ plasmid libraries. FtsK panel: XafT ${ }^{-}$plasmids conjugated in a strain harbouring dif1 at its natural locus; XafT panel: XafT ${ }^{+}$plasmids conjugated in a strain harbouring dif1 at the lacZ locus; FtsK \& XafT panel: XafT plasmids conjugated in a strain harbouring dif1 its natural locus. Mean and standard deviations of at least 3 independent assays. (B) 2D maps showing the relative integration frequency of the different possible $\mathrm{n}_{8}$ gtg sequences $\left(f_{\text {int }}\right)$. The scheme below the 2D maps indicates how specific horizontal and vertical coordinates are assigned to each degenerate motif. The positions of TLCФ attP and of the sequences most similar to dif1 are highlighted. (C) Butterfly plots showing the relative integration efficiency of the different possible $n_{8}$ gtg sequences. X-axis: number of changes from TLCФ attP (from 0 to 8$).+$ and - values indicate whether the changes render the site more similar to dif1 or not. The proportion of sequences falling in the - group is indicated. Their contribution to the global frequency of integration of the library is shown between brackets. A red, orange, cyan and blue colour code was assigned to the attP sites from the FtsK panel whose integration frequencies was higher than $10^{-6}, 10^{-7}, 510^{-8}$ or lower than $510^{-8}$, respectively. The corresponding sites in the XafT and Ftsk \& XafT panels were highlighted with the same colour code, with sites absent in the FtsK panel shown in grey. The difference between the mean frequency of integration of red motifs and the integration frequency of TLCФ attP is indicated in red. (D) Number of different $n_{8} g t g, n_{5} a_{2} n_{2} g$ and $\operatorname{tagn}_{8}$ sequences with an at frequencies higher than $10^{-6}$. The sequence logo shows the frequency of each base at the degenerate positions. 
A
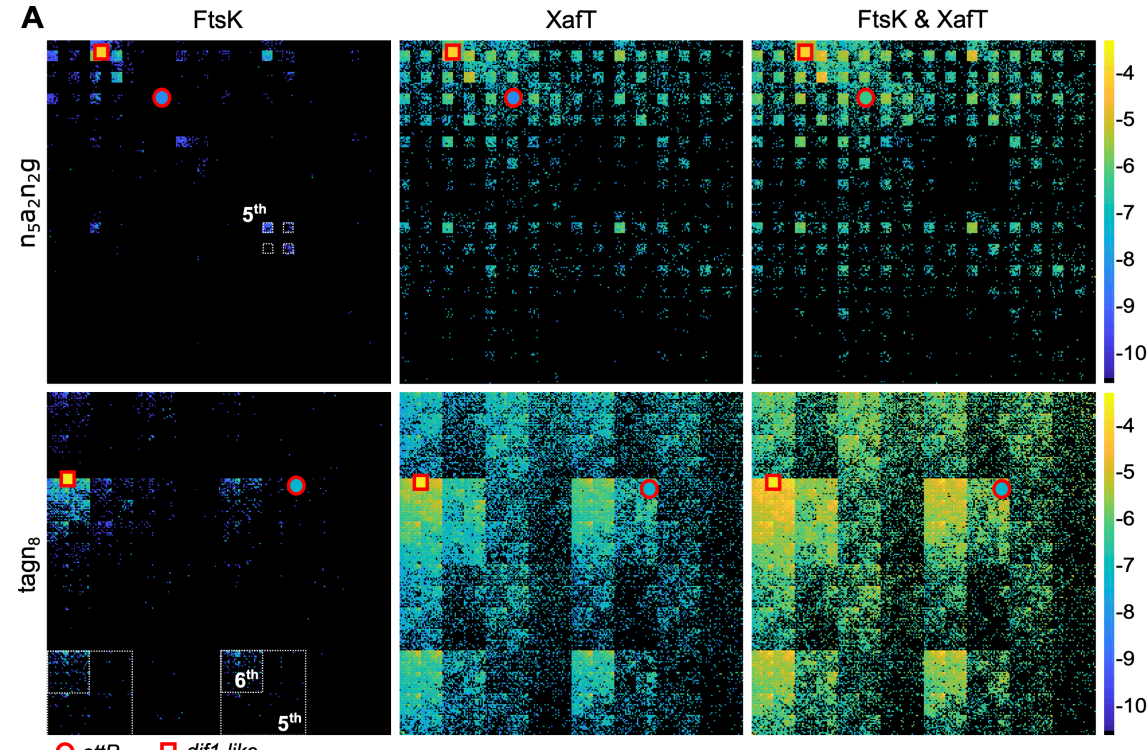

B
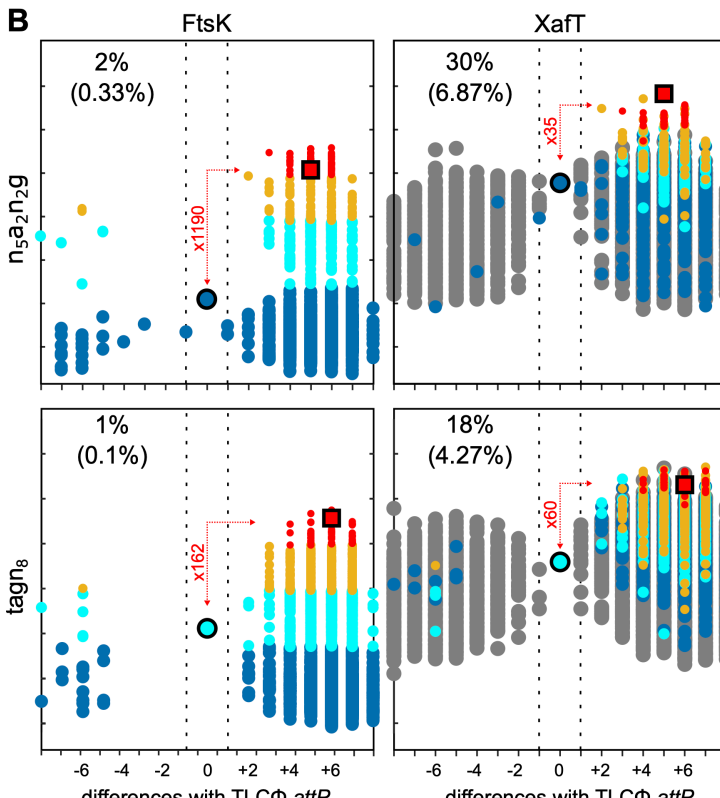

differences with TLC $\Phi$ attP

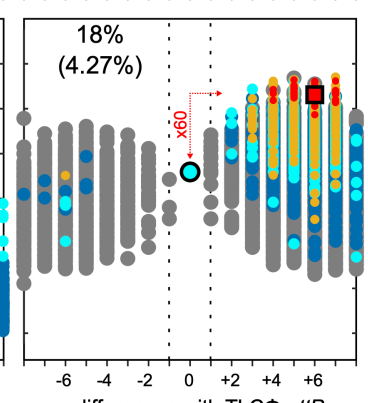

differences with TLCФ attP
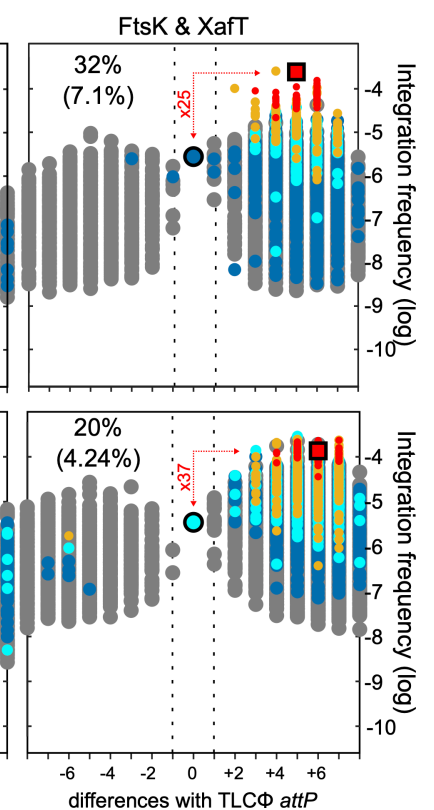

Supplementary Figure S3. Suicide mini-TLCФ plasmid integration. (A) 2D maps

Butterfly plot representation of the relative integration efficiency of the different possible sequences. Legend as in Figure 3.

\section{Mini-TLCФ plasmids with attP sites deviating from dif1 escape FtsK-driven excision}

An unusually long IMEX, the gonococcal genomic island (GGI), is integrated at the dif site of the chromosome of pathogenic Neisseria species $(29,30)$. It is flanked by a dif-like site with 4 non-canonical bp within the 6 outermost positions of the XerD-arm. The FtsK DNA translocase 
was shown to strip XerC and XerD bound to this site, thereby preventing GGI excision (24). It suggested the possibility that TLCФ attP was selected to allow XafT-driven integration while avoiding FtsK-driven excision events (Figure 1D). To explore this hypothesis, we monitored the stability of $\mathrm{XafT}^{+} \mathrm{n}_{8} \mathrm{gtg}$, tagn $\mathrm{n}_{8}$ and $\mathrm{n}_{5} \mathrm{a}_{2} \mathrm{n}_{2} \mathrm{~g}$ mini-TLCФ plasmids that were integrated at the dif1 locus. Production of XerC and XerD did not reduce the number of attP sites in the cell population (Figure 4A and S4, butterfly plots). However, there was a significant decrease in the proportion of attP sites from the red and orange categories (Figure 4A and S4, bar plots). Most importantly, the frequency of TLC $\Phi$ attP became higher than the frequency of the attP site that most resembled dif1 (Figure 4A and S4, butterfly plots).

To determine the relative contribution of FtsK and XafT to the observed excision events, we analysed the stability of $\mathrm{XafT}^{+} \mathrm{n}_{8}$ gtg plasmids integrated at the lacZ locus, where FtsK cannot act (Figure S2). After $42 \mathrm{~h}$ of growth, there were little changes in the frequency of the different attP sites (Figure 4B, butterfly plots) and in the relative proportion of the sites from the 5 colour code categories in the cell population (Figure 4B, bar plots). The proportion of the attP sites from the red category went down, but the decrease was much less important than the one observed at dif1 after only $19 \mathrm{~h}$ of growth. These results suggested that the expression of XafT was repressed after integration under normal laboratory growth conditions. In contrast, the proportion of attP sites from the red and orange categories was reduced by half after $42 \mathrm{~h}$ of growth in AKI, a medium designed to mimic the intestinal environment, which was previously shown to boost the expression of the genes from another V. cholerae IMEX, CTXФ (Figure 4C, bar plots, (31)). Furthermore, attP sites from the red, orange, cyan and blue categories represented less than $10 \%$ of the total sequence reads after $63 \mathrm{~h}$ of growth in AKI. In particular, there was a 100-fold decrease in the relative proportion of TLCФ attP (Figure 4C, butterfly plots). 
bioRxiv preprint doi: https://doi.org/10.1101/2021.09.09.459576; this version posted September 9, 2021. The copyright holder for this preprint (which was not certified by peer review) is the author/funder, who has granted bioRxiv a license to display the preprint in perpetuity. It is made available under aCC-BY-NC-ND 4.0 International license.

We conclude that FtsK is responsible for the excision of mini-TLCФ plasmids integrated at the dif1 locus under normal laboratory growth conditions. However, the excision frequency of most sites remained far lower than the $20 \%$ per generation excision frequency of a DNA cassette flanked by two dif1 sites (25). Thus, many other sites than TLCФ attP could have been selected for stability.

\section{A FtsK \& XafT}
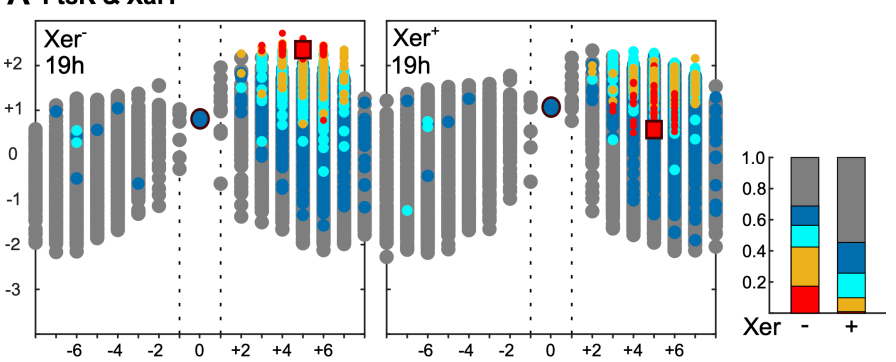

B XafT
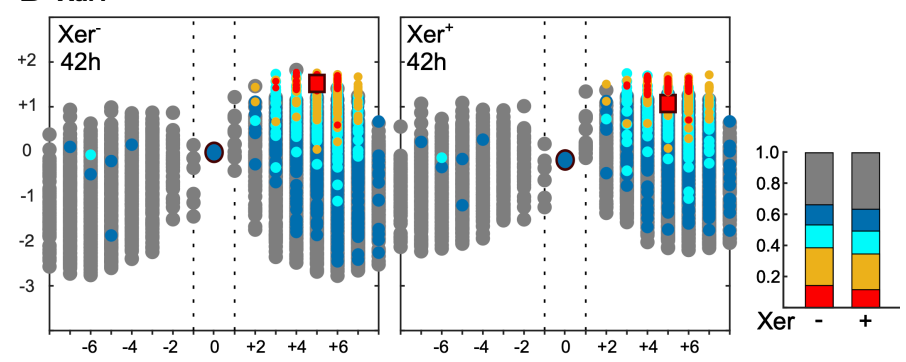

\section{XafT (AKI)}
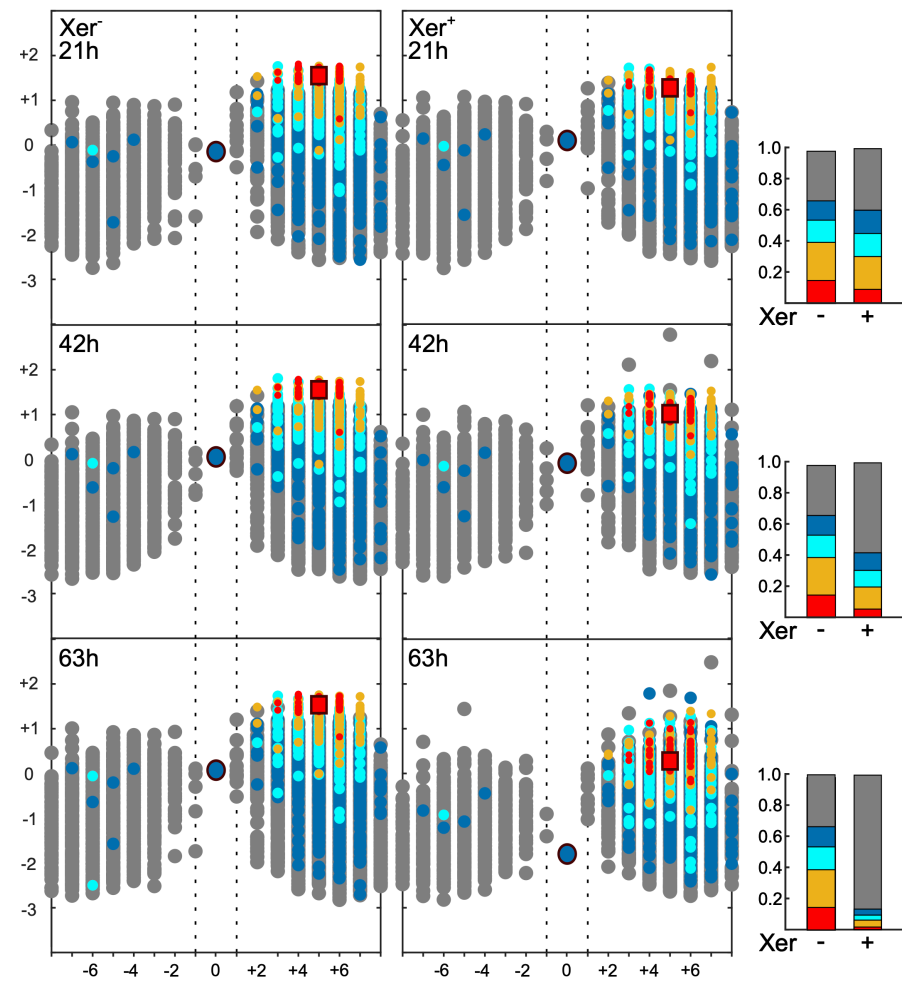

differences with TLCФ attP
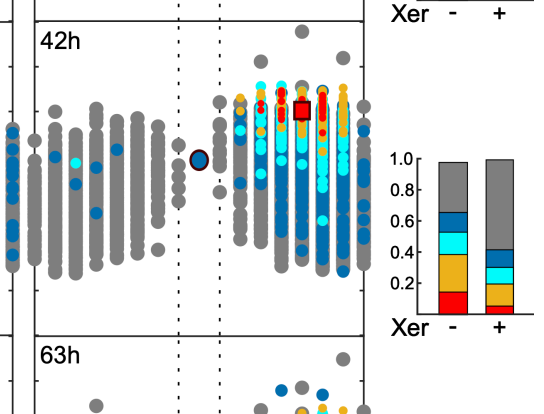
bioRxiv preprint doi: https://doi.org/10.1101/2021.09.09.459576; this version posted September $9,2021$. The copyright holder for this preprint (which was not certified by peer review) is the author/funder, who has granted bioRxiv a license to display the preprint in perpetuity. It is made available under aCC-BY-NC-ND 4.0 International license.

Figure 4. Relative stability of $\mathrm{XafT}^{+}$plasmids harbouring an attP site with the $\mathrm{n}_{8} \mathrm{gtg}$ motif. (A) Remaining plasmids at the natural dif1 locus after growth in LB. (B) Remaining plasmids integrated at the lacZ locus after growth in LB. (C) Remaining plasmids integrated at the lacZ locus after growth in AKI. Butterfly plots show the frequency of each motif as in Figure 3. Bar plots show the proportion of remaining motifs from the red, orange, cyan, blue and grey categories. Xer ${ }^{+/-}$indicate whether the inducer (L-arabinose) of XerC and XerD production was added to the growth medium or not.

\section{A FtsK \& XafT}

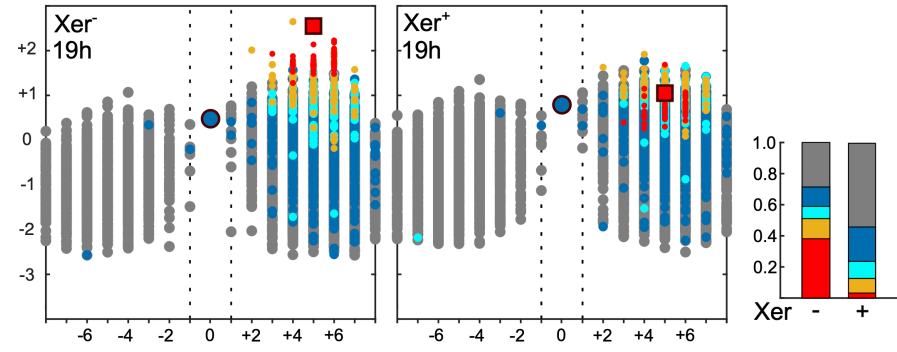

B FtsK \& XafT

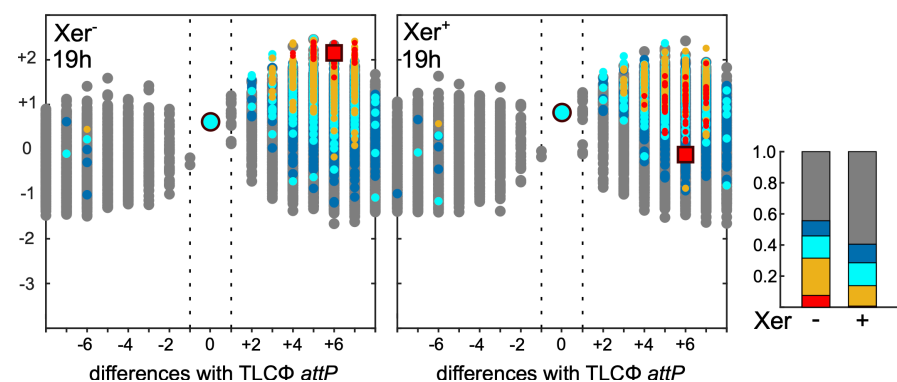

Supplementary Figure S4. Relative stability of XafT ${ }^{+}$plasmids. (A) Remaining $n_{5} a_{2} n_{3}$ g plasmids at the natural

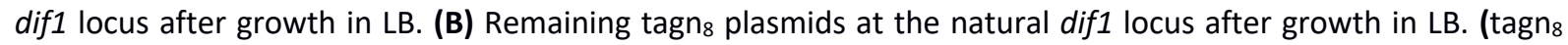
motif. Legend as in Figure 4.

\section{TLCФ attP/ dif1 synaptic complexes are rare and/or transient}

XafT activates XerD in trans via a direct interaction (Figure 5A, (23)). FtsKy also interacts with $\operatorname{XerD}(14,16,23,32)$. However, it acts in cis when FtsK is bound on the XerD side of one or the other of the two DNA duplexes engaged in the recombination complex (Figure 5A, (33, 34)). It suggested that FtsK translocation could dismantle TLCФ attP/ dif1 synaptic complexes before FtsK $\gamma$ had had the time to activate XerD, thereby preventing FtsK-driven integration events (Figure 5A, (24)). We reasoned that if this hypothesis was correct, a C-terminal fusion of FtsKy to XerD, which was previously shown to maximize the efficiency of reactions mediated by the E. coli and N. gonorrhoea Xer recombinases, should be able to promote the recombination of dif1 with TLCФ attP in vitro $(15,24)$. As a point of comparison, we reconstituted XafT-mediated 
reactions using XerC and XerD proteins and an N-terminal fusion of XafT to the maltose binding protein, MBP-XafT (23). We used a short 34-bp synthetic double-stranded DNA (dsDNA) dif1 fragment and longer dsDNA fragments containing either dif1 or TLCФ attP as substrates. To facilitate the differentiation of the $\mathrm{HJ}$ intermediate, the two crossover products and the substrate, the $5^{\prime}$ and $3^{\prime}$ sides of the bottom strand of the short dif1 substrate were labelled with cy5 and cy3 fluorescent dyes, respectively (Figure 5B). Incubation of the two dif1 substrates with XerC and XerD-FtsKY or with XerC, XerD and MBP-XafT yielded similar amounts of $\mathrm{HJs}$ and crossover products, suggesting that FtsK $\gamma$ promoted recombination as efficiently as XafT when it was fused to XerD (Figure 5C, left panel). Yet, incubation of the short labelled dif1 substrate and the TLCФ attP substrate with XerC and XerD-FtsK $\gamma$ yielded barely detectable amounts of HJs and crossover products, invalidating the idea that the low frequency of FtsKdriven integration events could be attributed to the translocase activity of FtsK (Figure 5C, right panel). In contrast, XerC, XerD and MBP-XafT mediated the formation of similarly high amounts of HJs and crossover products between the short labelled dif1 substrate and the long dif1 or TLCФ attP substrates (Figure 5B). We conclude that TLCФ attP/dif1 synaptic complexes are normally too rare and/or too transient to allow for FtsK $p$-driven activation of XerD. 


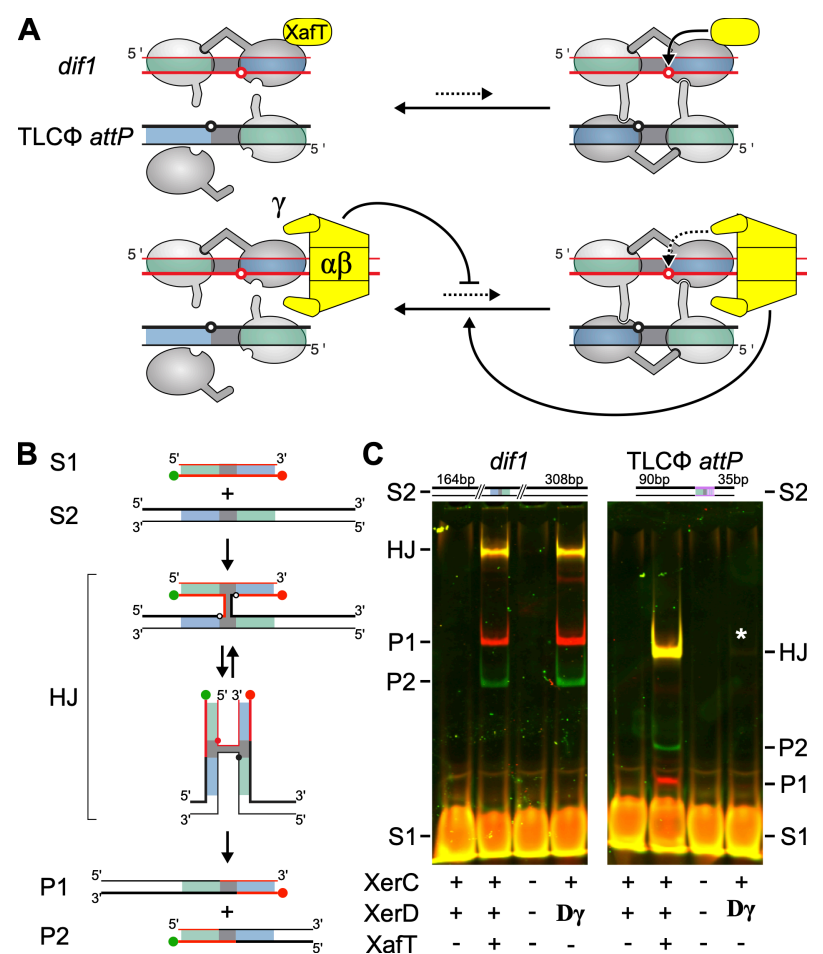

Figure 5. Relative efficiency of XafT- and FtsKY-driven Xer recombination reactions. (A) Scheme of the recombination substrates, the $\mathrm{HJ}$ recombination intermediate and the crossover products. Green ball: $3^{\prime} \mathrm{Cy} 3$ label; Red ball: 5' Cy5 label; Grey, Green and Blue rectangles: Central region, XerC-binding and XerD-binding arm, respectively. The DNA strands exchanges by XerC and XerD are depicted as thin and fat lines, respectively. (B) XafT- and FtsKY-promoted recombination reactions. Left panel: recombination of a short labelled dif1 substrate (S1) and a long non-labelled dif1 substrate (S2); Right panel: recombination of a short labelled dif1 substrate (S1) and a long non-labelled TLCФ attP substrate (S2). The S2 substrates are depicted above each gel images. +: $V$. cholerae XerD, $V$. cholerae XerD or MBP-XafT, as indicated; $\gamma$ : $V$. cholerae XerD-FtsK $\gamma$ fusion; - : mock buffer of the corresponding purified proteins. A white star indicates the presence of a faint $\mathrm{HJ}$ band.

\section{DISCUSSION}

Xer recombination takes place in a nucleoprotein complex consisting of two recombining sites and a pair of each of the two XerC and XerD recombinases (Figure 1). The complex is held together by the affinity of the recombinases to their DNA binding sites and cyclic interactions between their C-terminal domains. However, binding of XerC to dif is relatively weaker than binding of $\operatorname{XerD}(2,13,35)$. Those observations suggested that contacts between XerD and dif played a primary role in the assembly and stability of the recombination synapses $(2,13,35)$. In this regard, the efficiency with which TLCФ integrated in the primary chromosome of clinical and environmental clones of $V$. cholerae was surprising since the XerD-arm of TLCФ attP contains 8 bp difference from dif1, which almost completely abolishes XerD binding $(10,11)$. 
TLCФ relies on its own XerD activation factor for integration, XafT $(10,23)$. No other $V$. cholerae or TLCФ protein or sequence factors are required to promote a complete Xer recombination reaction between TLC $\Phi$ attP and dif1 in vitro (23). Those results suggested that XafT might itself open up the possibility to recombine TLCФ attP and dif1.

\section{XafT promotes the formation of and stabilizes synaptic complexes}

Our analysis of the influence of the XerD-arm of TLCD attP on the integration efficiency and stability of mini-TLCФ plasmids showed that FtsK only promotes the recombination of dif1 with a very small subset of the different 190528 TLCФ attP derived sites we studied, those that were the most similar to dif1 (Figure 3, S3, 4 and S4). In contrast, XafT promoted the recombination of dif1 with any of the 190528 attP sites that carried the same residues as dif1 at the $5^{\text {th }}$ position of the XerD-arm (Figure 3 and S3). In addition, it alleviated differences in the integration frequency of attP sites harbouring more or less canonical XerD-arms (Figure 3 and S3). Our results further indicated that FtsK did not perturb XafT-driven integration and excision events (Figure 3, S3 and 4). Finally, in vitro comparison of the efficiency with which XafT and FtsKy drove recombination reactions between two dif1 sites or between dif1 and TLCФ attP showed that TLCФ attP/ dif1 synaptic complexes are normally too rare and/or too transient to allow for FtsKy-driven activation of XerD (Figure 5). Taken together, those results suggest that XafT can promote the efficient recombination of dif1 with TLCФ attP-derived sites harbouring a non-canonical XerD-arm because it helps assemble and stabilize synaptic complexes. As XafT contains a dimerization domain and directly interacts with XerD, it is tempting to propose that it recruits a XerD recombinase in trans at the dif1 locus by forming a proteinaceous bridge with the XerD recombinase bound in cis (23). The resulting dif1/XerC/XerD/XafT/XerD complex could then be engaged in a larger nucleoprotein with an attP site solely bound by XerC via the interactions of recombinases. Thus, a limited amount of 
homology between the XerD-arm of the attP site and the canonical XerD binding site would be required to ensure the formation of recombination synapse.

\section{Role of the $\mathrm{G}$ base at $5^{\text {th }}$ innermost position of the XerD and XerC arms}

Our NGS data allowed us to revisit the importance of the nature of the different positions of the XerD arm of dif sites. Whether integration was driven by FtsK or by XafT, the mini-TLC $\Phi$ plasmids that integrated the most efficiently were those that harboured the most dif-like motifs (Figure 3 and S3). However, the 5 outermost positions of the XerD-arm were less constrained than the 6 innermost positions (Figure 3 and S3). In addition, the possibility for XafT-driven integration was mainly influenced by the presence of a guanine at the $5^{\text {th }}$ position of the top strand of the XerD-arm of the attP sites, with XafT promoting the integration of most if not all of the plasmids that carried this base (Figure 3 and S3). The nature of the $5^{\text {th }}$ position of the XerD-arm of the attP sites was also the most important for FtsK-driven integration (Figure 3 and S3). Correspondingly, it was previously observed in E. coli that a dif site carrying a single mutation at the $5^{\text {th }}$ position of the XerD-arm was less proficient than wildtype diffor Xer recombination (35). In addition, methylation interference analysis showed that E. coli XerD interacted with the guanine at the $5^{\text {th }}$ position of the top strand of its binding site (35).

It was recently reported that Bacillus subtilis and Staphylococcus aureus XerD unload bacterial SMC complexes (36). This activity is independent from XerC. It relies on the binding of XerD to additional chromosomal loci other than dif. Those loci harbour a dif-like site composed of a bona fide XerD binding site, a 5-6bp degenerate central region and the 5 innermost bp of the XerD binding site (36). Our results raise the possibility that those sites evolved to permit the cooperative binding of two XerD molecules. 
The specificity of binding of XerC and XerD is ensured by the bp composition of the $6^{\text {th }}, 7^{\text {th }}$ and $9^{\text {th }}$ to $11^{\text {th }}$ non-palindromic positions of their binding arms $(37,38)$. However, contacts between $E$. coli XerC and dif were shown to be mainly limited to the 7 innermost bp of its binding site (35). Correspondingly, only the innermost region of the XerC-arm of dif sites is conserved (Figure S1B). The 5 innermost positions of the XerC- and XerD-arms are identical (Figure 1B and S1B). As XerC and XerD are highly related (Figure S1A), it is reasonable to argue that the guanine at the $5^{\text {th }}$ position plays the same primary role for binding of XerC as it does for binding of XerD (Figure 3 and S3). This observation could explain the apparent inefficiency of integration of CTXФ in non-toxigenic environmental $V$. cholerae strains since both difA and the CTXФ attP contain a non-canonical T/A bp at the $5^{\text {th }}$ position of their XerC-arm (Figure S1B, (11)). By extrapolation, we propose that XafT can promote the integration of TLCФ into difA because it can stabilize the formation of recombination synapses between non-canonical sites (10).

Alternative Xer machineries composed of a single Xer recombinase are found in a few bacterial and archaeal species (39-41). The recombinases of those machineries are relatively distant from XerC and XerD (Figure S1A) and target sites that significantly deviate from classical dif sites (Figure S1B). A GC bp is also present at the 5th innermost position of the binding sites of alternative Xer recombinases (Figure S1B). It was shown to be important for the binding and activity of $H$. pylori XerH (39). However, this bp is inverted with respect to the GC bp of canonical XerC and XerD-arms (Figure S1B). X-ray structure analysis revealed that $H$. pylori XerH contacts the guanine of the bottom strand of its binding site with an arginine of its $\mathrm{N}$ terminal domain, R65 (39). In contrast, a model based on the crystal structure of E. coli XerD and the Catabolite Activator Protein-DNA complex suggested that XerD contacts the guanine of the top strand of its binding site with a conserved arginine of its C-terminal domain (R220 
in E. coli XerD and R224 in V. cholerae XerD, (42)). Those observations highlight the evolutionary distance between conventional and alternative Xer machineries.

\section{XerD-arm degeneracy prevents FtsK-driven Xer-mediated excision events}

The analysis of the stability of the XafT ${ }^{+} n_{8} g t g, n_{5} a_{2} n_{2} g$ and tagn $n_{8}$ plasmids that were integrated at the dif1 locus, which corresponds to the normal location of TLCФ in the genome of $V$. cholerae pathogenic clones of the current pandemic, revealed that the mini-TLCФ plasmids that were efficiently integrated by FtsK were also the less stable (Figure 4 and S4). In addition, all of the 190528 mini-TLCФ plasmids we studied, including those that harboured the attP sites that most resembled dif1 were far more stable that a DNA cassette flanked by two dif1 sites (Figure 4 and S4). Those observations lend support to the idea that the few non-canonical bp in the XerD-arm of the GGI and TLCФ attP sites serve to prevent their excision (24). Nevertheless, TLCФ can excise from its host genome (10). Our results suggest that those excision events are promoted by XafT independently of FtsK (Figure 4). They are extremely rare in normal laboratory growth conditions, but they can be increased in conditions that mimic cholera, which suggest that the production of XafT is tightly regulated (Figure 4).

\section{Altering the possibility for synapse formation is a major source of control}

The dimer resolution sites of multicopy plasmids exploiting Xer and the attachment sites of IMEX relying on the non-canonical XerC-first recombination pathway contain a few degenerate positions in the XerC and XerD arms, which could limit the efficiency of recombination (Figure S1B). Our results suggest that those mutations evolved to limit the formation of and/or stability of synaptic complexes, thereby preventing the formation of multimers by Xer recombination (43). In particular, there are two mutations in the inner region of the XerC-arm of the ColE1 dimer resolution site, cer, including one at the $5^{\text {th }}$ position, and there is a mutation at the $6^{\text {th }}$ position of its XerD-arm (Figure S1B). The efficiency of 
intramolecular dimer resolution events relies on accessory proteins that bind to accessory sequences flanking the core cer site, which bring together the core cer sites in a synaptic complex in a specific topological configuration $(22,44)$. It remains to be determined whether IMEX such as CTXФ or VGJФ simply rely on the amplification of their free form by replication to achieve efficient integration or whether unknown mechanisms favour the formation of recombination synapses between the host target dif site and their attP sites $(45,46)$. Future studies will also need to address the evolutionary pressures that seems to have set the noncanonical bp of each plasmid dimer resolution and IMEX attachment sites.

\section{ACKNOWLEDGEMENTS}

We thank James Provan, Raphaël Guérois and Virginia Lioy for helpful discussions. The work was supported by the ERC (FP7/2007-2013, grant number 28159), the ANR (grants 2016-CE120030-0 and 2018-CE12-0012-03) and the FRM (EQU202003010328).

\section{MATERIALS AND METHODS}

\section{Strains, plasmids and oligonucleotides}

Strains, plasmids and oligonucleotides are listed in Supplementary Tables S2, S3 and S4, respectively. Strains were built by natural transformation using appropriate selection markers and/or blue/white $\beta$-galactosidase screens. Plasmids pSM11 and pSM15 are pTLC8 and pSM12 derivatives, respectively (23). They were constructed by replacing the TLC $\Phi$ attp locus by the Bsal-ccdB-Bsal cassette of pFB5 (47) using Gibson assembly (48). Pools of n8gtg, tagn8 and n5a2g3 TLCФ attp-derived sites were built by primer extension of oligo 4069 annealed to degenerate 4066-4068 oligonucleotides. The recombination site libraries were inserted in pSM11 and pSM15, and cloned in FCV14. Transformation reactions were plated on $20 \mathrm{~cm}$ diameter petri dishes and repeated to obtain a total of about $10^{6}$ colonies. Plasmid libraries were then extracted from 1x10 10 FCV14 cells, and transformed into $\beta 2163$. 


\section{Integration assays}

V. cholerae recipients were grown to an OD600 of 0.3 in LB with $0.2 \%$ of L-arabinose (L-ara), to induce the Xer machinery. E. coli $\beta 2163$ donors were grown to an OD600 of 0.6 in LB supplemented with $0.3 \mathrm{mM}$ of Dap, and mixed with recipients at a 1:10 ratio. Donor and recipient cells were incubated for $3 \mathrm{~h}$ on LB agar plates supplemented with Dap and L-ara, resuspended in LB supplemented with $0.2 \%$ of L-ara and incubated for an additional $1 \mathrm{~h}$. Conjugants were selected for the plasmid antibiotic resistance and Dap autotrophy. In the case of the degenerate plasmid libraries, several conjugations were performed for each integration assay to ensure the recovery of $\sim 10^{6}$ clones.

\section{Stability assays}

To observe the evolution of the different attP classes during the proliferation FtsK and XafT, integration libraries were incubated without any selection pressure for the maintenance of the integrated elements. Fresh LB was inoculated with $\sim 1 \times 10^{9}$ cells from the integration libraries, with the addition of arabinose $0.2 \%$ for the expression of XerD and XerC.

\section{NGS analysis}

Plasmid attP sites were amplified by performing 17 PCR cycles on 50-100 ng of plasmid library gDNA with an equimolar mix of 4103-4105 P5 and 4178-4180/4246-4248 P7 primers. The products were purified from the P5 and P7 primers the double selection with AMPure. NextSeq reads were trimmed with Cutadapt (version 1.17). For the 2D-maps, different $[x y]$ coordinates were assigned to each nucleotide for the 65536 possible motifs as follows: the $[x, y]$ coordinates were initially set to [0 0]. Then, $[1 / 2 n-1,1 / 2 n-1],[-1 / 2 n-1 / 2 n-1],[-1 / 2 n-1$, $-1 / 2 n-1]$ or $[1 / 2 n-1,-1 / 2 n-1]$ were added to $[x, y]$ for each $n$ position of the degenerate motif if the base of the recombination site was $A, T, G$ or $C$, respectively.

\section{In vitro recombination assays}


bioRxiv preprint doi: https://doi.org/10.1101/2021.09.09.459576; this version posted September $9,2021$. The copyright holder for this preprint (which was not certified by peer review) is the author/funder, who has granted bioRxiv a license to display the preprint in perpetuity. It is made available under aCC-BY-NC-ND 4.0 International license.

Proteins were purified and as In vitro recombination assays were performed as described in (23).

\section{REFERENCES}

1. C. Midonet, F.-X. Barre, Xer Site-Specific Recombination: Promoting Vertical and Horizontal Transmission of Genetic Information. Microbiol. Spectr. 2 (2014).

2. G. Blakely, et al., Two related recombinases are required for site-specific recombination at dif and cer in $\mathrm{E}$. coli K12. Cell 75, 351-361 (1993).

3. S. D. Colloms, P. Sykora, G. Szatmari, D. J. Sherratt, Recombination at ColE1 cer requires the Escherichia coli xerC gene product, a member of the lambda integrase family of site-specific recombinases. J Bacteriol 172, 6973-80 (1990).

4. B. Das, E. Martínez, C. Midonet, F.-X. Barre, Integrative mobile elements exploiting Xer recombination. Trends Microbiol. 21, 23-30 (2013).

5. P. Balalovski, I. Grainge, Mobilization of pdif modules in Acinetobacter: A novel mechanism for antibiotic resistance gene shuffling? Mol. Microbiol. (2020) https:/doi.org/10.1111/mmi.14563.

6. M. K. Waldor, J. J. Mekalanos, Lysogenic conversion by a filamentous phage encoding cholera toxin. Science 272, 1910-1914 (1996).

7. K. E. Huber, M. K. Waldor, Filamentous phage integration requires the host recombinases XerC and XerD. Nature 417, 656-659 (2002).

8. B. Das, J. Bischerour, M.-E. Val, F.-X. Barre, Molecular keys of the tropism of integration of the cholera toxin phage. Proc. Natl. Acad. Sci. U. S. A. 107, 4377-4382 (2010).

9. B. Das, J. Bischerour, F.-X. Barre, VGJphi integration and excision mechanisms contribute to the genetic diversity of Vibrio cholerae epidemic strains. Proc. Natl. Acad. Sci. U. S. A. 108, 2516-2521 (2011).

10. C. Midonet, B. Das, E. Paly, F.-X. Barre, XerD-mediated FtsK-independent integration of TLC $\varphi$ into the Vibrio cholerae genome. Proc. Natl. Acad. Sci. U. S. A. 111, 16848-16853 (2014).

11. F. Hassan, M. Kamruzzaman, J. J. Mekalanos, S. M. Faruque, Satellite phage TLC $\phi$ enables toxigenic conversion by CTX phage through dif site alteration. Nature 467, 982-985 (2010).

12. L. Aussel, et al., FtsK Is a DNA motor protein that activates chromosome dimer resolution by switching the catalytic state of the XerC and XerD recombinases. Cell 108, 195-205 (2002).

13. M.-E. Val, et al., FtsK-dependent dimer resolution on multiple chromosomes in the pathogen Vibrio cholerae. PLoS Genet. 4, e1000201 (2008).

14. J. Yates, M. Aroyo, D. J. Sherratt, F.-X. Barre, Species specificity in the activation of Xer recombination at dif by FtsK. Mol. Microbiol. 49, 241-249 (2003).

15. I. Grainge, C. Lesterlin, D. J. Sherratt, Activation of XerCD-dif recombination by the FtsK DNA translocase. Nucleic Acids Res 39, 5140-8 (2011).

16. J. Yates, et al., Dissection of a functional interaction between the DNA translocase, FtsK, and the XerD recombinase. Mol Microbiol 59, 1754-66 (2006). 
17. S. Bigot, et al., KOPS: DNA motifs that control E. coli chromosome segregation by orienting the FtsK translocase. EMBO J. 24, 3770-3780 (2005).

18. S. P. Kennedy, F. Chevalier, F.-X. Barre, Delayed activation of Xer recombination at dif by FtsK during septum assembly in Escherichia coli. Mol. Microbiol. 68, 1018-1028 (2008).

19. F. X. Barre, et al., FtsK functions in the processing of a Holliday junction intermediate during bacterial chromosome segregation. Genes Dev. 14, 2976-2988 (2000).

20. F. Cornet, J. Louarn, J. Patte, J. M. Louarn, Restriction of the activity of the recombination site dif to a small zone of the Escherichia coli chromosome. Genes Dev 10, 1152-61 (1996).

21. M.-E. Val, et al., The single-stranded genome of phage CTX is the form used for integration into the genome of Vibrio cholerae. Mol. Cell 19, 559-566 (2005).

22. S. D. Colloms, J. Bath, D. J. Sherratt, Topological selectivity in Xer site-specific recombination. Cell 88, 855864 (1997).

23. C. Midonet, S. Miele, E. Paly, R. Guerois, F.-X. Barre, The TLCФ satellite phage harbors a Xer recombination activation factor. Proc. Natl. Acad. Sci. 116, 18391-18396 (2019).

24. F. Fournes, et al., FtsK translocation permits discrimination between an endogenous and an imported Xer/dif recombination complex. Proc. Natl. Acad. Sci. U. S. A. 113, 7882-7887 (2016).

25. E. Galli, C. Midonet, E. Paly, F.-X. Barre, Fast growth conditions uncouple the final stages of chromosome segregation and cell division in Escherichia coli. PLoS Genet. 13, e1006702 (2017).

26. E. Espinosa, E. Paly, F.-X. Barre, High-Resolution Whole-Genome Analysis of Sister-Chromatid Contacts. Mol. Cell 79, 857-869.e3 (2020).

27. G. Demarre, et al., Differential management of the replication terminus regions of the two Vibrio cholerae chromosomes during cell division. PLoS Genet. 10, e1004557 (2014).

28. A. David, et al., The two Cis-acting sites, parS1 and oriC1, contribute to the longitudinal organisation of Vibrio cholerae chromosome I. PLoS Genet. 10, e1004448 (2014).

29. J. P. Dillard, H. S. Seifert, A variable genetic island specific for Neisseria gonorrhoeae is involved in providing DNA for natural transformation and is found more often in disseminated infection isolates. Mol Microbiol 41, 263-77 (2001).

30. N. M. Domínguez, K. T. Hackett, J. P. Dillard, XerCD-Mediated Site-Specific Recombination Leads to Loss of the 57-Kilobase Gonococcal Genetic Island. J. Bacteriol. 193, 377-388 (2011).

31. M. Iwanaga, K. Yamamoto, New medium for the production of cholera toxin by Vibrio cholerae O1 biotype El Tor. J. Clin. Microbiol. 22, 405-408 (1985).

32. A. N. Keller, et al., Activation of Xer-recombination at dif: structural basis of the FtsKy-XerD interaction. Sci. Rep. 6, 33357 (2016).

33. T. H. Massey, L. Aussel, F.-X. Barre, D. J. Sherratt, Asymmetric activation of Xer site-specific recombination by FtsK. EMBO Rep. 5, 399-404 (2004).

34. L. Bonné, S. Bigot, F. Chevalier, J.-F. Allemand, F.-X. Barre, Asymmetric DNA requirements in Xer recombination activation by FtsK. Nucleic Acids Res. 37, 2371-2380 (2009).

35. G. W. Blakely, D. J. Sherratt, Interactions of the site-specific recombinases XerC and XerD with the recombination site dif. Nucleic Acids Res. 22, 5613-5620 (1994). 
bioRxiv preprint doi: https://doi.org/10.1101/2021.09.09.459576; this version posted September $9,2021$. The copyright holder for this preprint (which was not certified by peer review) is the author/funder, who has granted bioRxiv a license to display the preprint in perpetuity. It is made available under aCC-BY-NC-ND 4.0 International license.

36. X. Karaboja, et al., XerD unloads bacterial SMC complexes at the replication terminus. Mol. Cell 81, 756766.e8 (2021).

37. G. Blakely, D. Sherratt, Determinants of selectivity in Xer site-specific recombination. Genes Dev. 10, 762773 (1996).

38. F. Hayes, D. J. Sherratt, Recombinase binding specificity at the chromosome dimer resolution site dif of Escherichia coli. J Mol Biol 266, 525-37 (1997).

39. A. Bebel, E. Karaca, B. Kumar, W. M. Stark, O. Barabas, Structural snapshots of Xer recombination reveal activation by synaptic complex remodeling and DNA bending. eLife $\mathbf{5}$.

40. P. Le Bourgeois, et al., The Unconventional Xer Recombination Machinery of Streptococci/Lactococci. PLoS Genet 3, e117 (2007).

41. I. G. Duggin, N. Dubarry, S. D. Bell, Replication termination and chromosome dimer resolution in the archaeon Sulfolobus solfataricus. EMBO J. 30, 145-153 (2011).

42. H. S. Subramanya, et al., Crystal structure of the site-specific recombinase, XerD. EMBO J 16, 5178-87 (1997).

43. D. K. Summers, C. W. Beton, H. L. Withers, Multicopy plasmid instability: the dimer catastrophe hypothesis. Mol Microbiol 8, 1031-8 (1993).

44. M. Bregu, D. J. Sherratt, S. D. Colloms, Accessory factors determine the order of strand exchange in Xer recombination at psi. EMBO J. 21, 3888-3897 (2002).

45. J. Bischerour, C. Spangenberg, F.-X. Barre, Holliday junction affinity of the base excision repair factor Endo III contributes to cholera toxin phage integration. EMBO J. 31, 3757-3767 (2012).

46. E. Martínez, E. Paly, F.-X. Barre, CTX $\phi$ Replication Depends on the Histone-Like HU Protein and the UvrD Helicase. PLoS Genet. 11, e1005256 (2015).

47. C. Engler, R. Kandzia, S. Marillonnet, A one pot, one step, precision cloning method with high throughput capability. PloS One 3, e3647 (2008).

48. D. G. Gibson, et al., Enzymatic assembly of DNA molecules up to several hundred kilobases. Nat. Methods 6, 343-345 (2009).

49. E. Galli, et al., Cell division licensing in the multi-chromosomal Vibrio cholerae bacterium. Nat. Microbiol. 1, 16094 (2016).

50. G. Demarre, et al., A new family of mobilizable suicide plasmids based on broad host range R388 plasmid (IncW) and RP4 plasmid (IncPa) conjugative machineries and their cognate Escherichia coli host strains. Res. Microbiol. 156, 245-255 (2005).

51. M.-L. Diebold-Durand, F. Bürmann, S. Gruber, "High-Throughput Allelic Replacement Screening in Bacillus subtilis" in SMC Complexes: Methods and Protocols, Methods in Molecular Biology., A. Badrinarayanan, Ed. (Springer, 2019), pp. 49-61. 


\section{FIGURE LEGENDS}

\section{Figure 1. Xer recombination.}

(A) Toxigenic conversion of $V$. cholerae. Env. and Clinic. Chr1: primary chromosome of environmental and clinical V. cholerae. (B) TLCФ attP and V. cholerae dif1 sequence. Grey: central region; Green: XerC arm; Blue: XerD arm; Pink: TLC $\Phi$ non-canonical bp; white and black triangles: XerC and XerD cleavage sites. (C) Xer recombination pathways. Black arrows: conventional recombination pathway; Red arrows: non-conventional asymmetric recombination pathway. The XerD and XerC cleavage points are depicted by empty and filled disks. (D) TLCФ excision/integration balance. Left: FtsK dismantles the excision complex when it translocates on the prophage DNA; Right: the non-canonical XerD-arm of TLCФ attP abolishes binding of XerD.

Figure 2. Parallel monitoring of integration and stability.

(A) Mini-TLCФ plasmid libraries. Top panel: top strand of the degenerate attP motifs. Legend as in Figure 1. Middle panel: scheme of $\mathrm{XafT}^{+}$and XafT-mini-TLCФ plasmids. Pink: TLCФ DNA; Grey: plasmid DNA. Yellow rectangle: xafT gene; sawed lines: stop mutation; T: RP4 transfer origin; 6: pir-dependent replication origin; cat: chloramphenicol resistance gene. Plasmidspecific P5 and TLCФ-specific P7 adaptor primers used for next generation sequencing (NGS) are indicated by grey and pink arrows, respectively. Bottom panel: distribution frequency of the motifs. (B) Integration and excision assays. On and off: growth in the presence or absence of arabinose. $\pi$ : R6K replication initiator; dap: diaminopimelic acid; $\mathrm{Cm}$ : chloramphenicol; Xgal: X-gal; Z $\alpha$ and ZB: E. coli lacZ gene. The lacZ $\alpha$-specific $\mathrm{P} 5$ adaptor primer is indicated by a black arrow.

Figure 3. Suicide mini-TLCФ plasmid integration. 
(A) Integration frequencies of mini-TLCФ plasmids harbouring dif1 or TLCФ attP, and global integration frequencies $(f)$ of $\mathrm{n}_{8} g t g, \mathrm{n}_{5} \mathrm{a}_{2} \mathrm{n}_{2} \mathrm{~g}$ and tagn $\mathrm{n}_{8}$ plasmid libraries. FtsK panel: XafTplasmids conjugated in a strain harbouring dif1 at its natural locus; XafT panel: XafT ${ }^{+}$plasmids conjugated in a strain harbouring dif1 at the lacZ locus; FtsK \& XafT panel: XafT ${ }^{+}$plasmids conjugated in a strain harbouring dif1 its natural locus. Mean and standard deviations of at least 3 independent assays. (B) 2D maps showing the relative integration frequency of the different possible $\mathrm{n}_{8} \mathrm{gtg}$ sequences $\left(f_{\text {int }}\right)$. The scheme below the $2 \mathrm{D}$ maps indicates how specific horizontal and vertical coordinates are assigned to each degenerate motif. The positions of TLCФ attP and of the sequences most similar to dif1 are highlighted. (C) Butterfly plots showing the relative integration efficiency of the different possible $\mathrm{n}_{8}$ gtg sequences. $\mathrm{X}$ axis: number of changes from TLCФ attP (from 0 to 8). + and - values indicate whether the changes render the site more similar to dif1 or not. The proportion of sequences falling in the - group is indicated. Their contribution to the global frequency of integration of the library is shown between brackets. A red, orange, cyan and blue colour code was assigned to the attP sites from the FtsK panel whose integration frequencies was higher than $10^{-6}, 10^{-7}, 510^{-8}$ or lower than $510^{-8}$, respectively. The corresponding sites in the XafT and Ftsk \& XafT panels were highlighted with the same colour code, with sites absent in the FtsK panel shown in grey. The difference between the mean frequency of integration of red motifs and the integration frequency of TLCФ attP is indicated in red. (D) Number of different $n_{8} g t g, n_{5} a_{2} n_{2} g$ and tagn sequences with an at frequencies higher than $10^{-6}$. The sequence logo shows the frequency of each base at the degenerate positions.

Figure 4. Relative stability of XafT+ plasmids. 
(A) Remaining $\mathrm{n}_{8}$ gtg plasmids at the natural dif1 locus after growth in LB. (B) Remaining plasmids integrated at the lacZ locus after growth in LB. (C) Remaining $n_{8}$ gtg plasmids integrated at the lacZ locus after growth in AKI. Butterfly plots show the frequency of each motif as in Figure 3. Bar plots show the proportion of remaining motifs from the red, orange, cyan, blue and grey categories. $\mathrm{Xer}^{+/-}$indicate whether the inducer (L-arabinose) of XerC and XerD production was added to the growth medium or not.

\section{Figure 5. Relative efficiency of XafT- and FtsKY-driven Xer recombination reactions.}

(A) Scheme of the recombination substrates, the $\mathrm{HJ}$ recombination intermediate and the crossover products. Green ball: 3' Cy3 label; Red ball: 5' Cy5 label; Grey, Green and Blue rectangles: Central region, XerC-binding and XerD-binding arm, respectively . The DNA strands exchanges by XerC and XerD are depicted as thin and fat lines, respectively. (B) XafT- and FtsKy-promoted recombination reactions. Left panel: recombination of a short labelled dif1 substrate (S1) and a long non-labelled dif1 substrate (S2); Right panel: recombination of a short labelled dif1 substrate (S1) and a long non-labelled TLCФ attP substrate (S2). The S2 substrates are depicted above each gel images. +: V. cholerae XerD, V. cholerae XerD or MBPXafT, as indicated; $\vee$ : $V$. cholerae XerD-FtsK $\gamma$ fusion; - : mock buffer of the corresponding purified proteins. A white star indicates the presence of a faint $\mathrm{HJ}$ band. 


\section{SUPPLEMENTARY FIGURE LEGENDS}

\section{Supplementary Figure 1. Xer recombination.}

(A) Phylogeny of Xer recombinases. Grey, Green, Blue, Orange, Pink and Magenta sectors: Cre (outgroup), XerC, XerD, XerH, XerS, XerA and Intl families, respectively. Vc: V. cholerae; Hp: Helicobacter pylori; LI: Lactoccocus lactis; Pa: Pyrococcus abessi. (B) Sequence of the top strand of bacterial dif sites and of Xer recombination sites harboured by typical mobile genetic elements. The top strand is the site cleaved by XerC during recombination. Grey: central region; Green, Blue, Magenta, Orange and Pink: XerC-, XerD-, Pa XerA-, Hp XerH- and LI XerSbinding arms, respectively. $\nu$-proteo: $\gamma$-proteobacteria dif consensus. ColE1 cer: core of the ColE1 plasmid dimer resolution site. Attachment site bases homologous to the host dif sequence are indicated by a hyphen. CTXФ attP is the stem of a folded hairpin, with $12 \mathrm{nt}$ on the top strand of its central region and 7 on its bottom strand. Nm: Neisseria meningitidis.

Figure S2. Parallel monitoring of integration and stability.

Scheme of the V. cholerae reporter strains. White circle: chromosome 1 replication origin; red, blue and black crosses: IMEX, xerD and lacZ deletions, respectively; black rectangle: E. coli lacZ $\alpha$-dif1-lacZ $\beta$ gene; light and dark grey rectangles: synthetic xerC and xerD operon under the control of the arabinose promoter (Para). Grey and yellow shadings depict the subcellular region and timing of activity of FtsK during the cell cycle.

\section{Supplementary Figure 3. Integration of suicide mini-TLCФ plasmids.}

(A) 2D maps representation of the relative integration efficiency of the different possible sequences. (B) Butterfly plot representation of the relative integration efficiency of the different possible sequences. Legend as in Figure 3.

\section{Supplementary Figure 4. Relative stability of $\mathrm{XafT}^{+}$plasmids.}


bioRxiv preprint doi: https://doi.org/10.1101/2021.09.09.459576; this version posted September 9,2021 . The copyright holder for this

preprint (which was not certified by peer review) is the author/funder, who has granted bioRxiv a license to display the preprint in perpetuity. It is made available under aCC-BY-NC-ND 4.0 International license.

(A) Remaining n5a2n3g plasmids at the natural dif1 locus after growth in LB. (B) Remaining

tagn8 plasmids at the natural dif1 locus after growth in LB. (tagn8 motif. Legend as in Figure

4.

\section{SUPPLEMENTARY TABLES}

Table S1. NGS data.

\begin{tabular}{|c|c|c|c|c|c|c|c|c|}
\hline \multirow[b]{2}{*}{$\begin{array}{c}\text { Degenerate } \\
\text { pool }\end{array}$} & \multirow[b]{2}{*}{$\begin{array}{l}\text { XeD activation } \\
\text { factor }\end{array}$} & \multirow[b]{2}{*}{ Sequence library } & \multirow[b]{2}{*}{$\begin{array}{c}\text { Number of } \\
\text { reads }\end{array}$} & \multirow[b]{2}{*}{$\begin{array}{c}\text { Expected } \\
\text { copy } \\
\text { number }\end{array}$} & \multirow[b]{2}{*}{$\begin{array}{l}\text { Absent } \\
\text { motifs }\end{array}$} & \multirow[b]{2}{*}{$\begin{array}{c}\% \text { present } \\
\text { motifs }\end{array}$} & \multicolumn{2}{|c|}{ Combined pool } \\
\hline & & & & & & & $\begin{array}{l}\text { Absent } \\
\text { motifs }\end{array}$ & $\begin{array}{c}\% \text { present } \\
\text { motifs }\end{array}$ \\
\hline n8gtg & XafT & Plasmid & 3014513 & 46 & 87 & $99,9 \%$ & \multirow{3}{*}{270} & \multirow{3}{*}{$99,9 \%$} \\
\hline tagn8 & XafT & Plasmid & 1375803 & 21 & 146 & $99,8 \%$ & & \\
\hline$n 5 a 2 n 3 g$ & XafT & Plasmid & 1003332 & 15 & 165 & $99,7 \%$ & & \\
\hline n8gtg & - & Plasmid & 1500796 & 23 & 318 & $99,5 \%$ & \multirow{3}{*}{425} & \multirow{3}{*}{$99,8 \%$} \\
\hline tagn8 & - & Plasmid & 1443196 & 22 & 62 & $99,9 \%$ & & \\
\hline$n 5 a 2 n 3 g$ & - & Plasmid & 1433248 & 22 & 126 & $99,8 \%$ & & \\
\hline n8gtg & FtsK & Integration & 865170 & 13 & 64116 & $2,2 \%$ & \multirow{4}{*}{185793} & \multirow{3}{*}{$2,5 \%$} \\
\hline tagn8 & FtsK & Integration & 784541 & 12 & 63381 & $3,3 \%$ & & \\
\hline$n 5 a 2 n 3 g$ & FtsK & Integration & 981722 & 15 & 64324 & $1,8 \%$ & & \\
\hline n8gtg & XafT & Integration & 2083611 & 32 & 55038 & $16,0 \%$ & & \multirow{3}{*}{$25,0 \%$} \\
\hline $\operatorname{tagn} 8$ & XafT & Integration & 1684057 & 26 & 35252 & $46,2 \%$ & \multirow[t]{3}{*}{142984} & \\
\hline$n 5 a 2 n 3 g$ & XafT & Integration & 891750 & 14 & 57896 & $11,7 \%$ & & \\
\hline n8gtg & FtsK \& XafT & Integration & 1469756 & 22 & 53936 & $17,7 \%$ & & \multirow{3}{*}{$27,9 \%$} \\
\hline tagn8 & FtsK \& XafT & Integration & 1166802 & 18 & 33214 & $49,3 \%$ & \multirow[t]{2}{*}{137400} & \\
\hline n5a2n3g & FtsK \& XafT & Integration & 1022000 & 16 & 55223 & $15,7 \%$ & & \\
\hline n8gtg & FtsK \& XafT & Excision Xer- 19h & 1188318 & 18 & 53784 & $17,9 \%$ & \multirow{3}{*}{137606} & \multirow{3}{*}{$27,8 \%$} \\
\hline tagn8 & FtsK \& XafT & Excision Xer- 19h & 1249627 & 19 & 33001 & $49,6 \%$ & & \\
\hline $\mathrm{n} 5 \mathrm{a} 2 \mathrm{n} 3 \mathrm{~g}$ & FtsK \& XafT & Excision Xer- 19h & 1202064 & 18 & 55845 & $14,8 \%$ & & \\
\hline n8gtg & FtsK \& XafT & Excision Xer+ 19h & 1575885 & 24 & 52833 & $19,4 \%$ & \multirow{3}{*}{135158} & \multirow{3}{*}{$29,1 \%$} \\
\hline tagn8 & FtsK \& XafT & Excision Xer+ 19h & 1274840 & 19 & 32094 & $51,0 \%$ & & \\
\hline$n 5 a 2 n 3 g$ & FtsK \& XafT & Excision Xer+ 19h & 771612 & 12 & 55160 & $15,8 \%$ & & \\
\hline n8gtg & XafT & Excision Xer- $42 \mathrm{~h}$ & 1542591 & 24 & 55548 & $15,2 \%$ & - & - \\
\hline n8gtg & XafT & Excision Xer+ $42 \mathrm{~h}$ & 473362 & 7 & 56523 & $13,8 \%$ & - & - \\
\hline n8gtg & XafT & Excision Xer- 21h & 1082288 & 17 & 55903 & $14,7 \%$ & - & - \\
\hline n8gtg & XafT & Excision Xer- 42h & 1334926 & 20 & 55811 & $14,8 \%$ & - & - \\
\hline n8gtg & XafT & Excision Xer- 63h & 1518529 & 23 & 55639 & $15,1 \%$ & - & - \\
\hline n8gtg & XafT & Excision Xer+ $21 \mathrm{~h}$ & 1085199 & 17 & 55698 & $15,0 \%$ & - & - \\
\hline n8gtg & XafT & Excision Xer+ $42 \mathrm{~h}$ & 1075984 & 16 & 56049 & $14,5 \%$ & - & - \\
\hline n8gtg & XafT & Excision Xer+ 63h & 1179951 & 18 & 58085 & $11,4 \%$ & - & - \\
\hline
\end{tabular}

Table S2. Strain list

\begin{tabular}{|c|c|c|}
\hline & Genotype & Reference \\
\hline CMV30 & $\begin{array}{l}\text { N1696 ChapR } \Delta x e r D:: \text { sh ble (ZeoR) xerC::paraXerCD-SpecR } \Delta \text { lacZ } \\
\text { dif1-prophages::EclacZa-dif1-lacZb }\end{array}$ & (49) \\
\hline EPV369 & $\begin{array}{l}\text { N16961 ChapR xerC::paraXerCD-SpecR } \Delta / a c Z:: l a c Z \alpha \text {-dif1-lacZB } \Delta \text { dif1- } \\
\text { prophages } \Delta x e r D:: \text { sh ble (ZeoR) }\end{array}$ & This study \\
\hline EPV366 & $\begin{array}{l}\text { N16961 ChapR xerC::paraXerCD-SpecR } \Delta / a c Z:: \text { lacZ } \alpha \text {-dif1-lacZ } \beta \text { @ } \\
\text { lacZ } \Delta \text { dif1-prophages }\end{array}$ & This study \\
\hline FCV14 & E. coli xerC::KanR DH5 $\alpha$ producing the R6K $\pi$ protein & $\begin{array}{l}\text { Laboratory } \\
\text { collection }\end{array}$ \\
\hline$\beta 2163$ & E. coli (F-) RP4-2-Tc::Mu $\Delta$ dapA::(erm-pir) $\left[\mathrm{Km}^{\mathrm{R}} \mathrm{Em}^{\mathrm{R}}\right]$ & $(50)$ \\
\hline
\end{tabular}


bioRxiv preprint doi: https://doi.org/10.1101/2021.09.09.459576; this version posted September $9,2021$. The copyright holder for this preprint (which was not certified by peer review) is the author/funder, who has granted bioRxiv a license to display the preprint in perpetuity. It is made available under aCC-BY-NC-ND 4.0 International license.

Table S3. Plasmid list.

\begin{tabular}{|c|c|c|}
\hline Plasmids & Properties & Reference \\
\hline $\begin{array}{l}\text { pTLC8 } \\
\text { (pCM33) }\end{array}$ & pTLC derived vector lacking all TLCФ nucleotides from 325 to 4137 & (1) \\
\hline $\begin{array}{l}\text { pTLC10 } \\
\quad(\text { pCM24) }\end{array}$ & pTLC derived vector with a stop mutation in VC1465. & $\begin{array}{c}\text { Midonet et } \\
\text { al., } \\
\text { 2014) }\end{array}$ \\
\hline $\begin{array}{l}\text { pTLC-Cri } \\
\quad(p B S 90)\end{array}$ & R6K $y$ ori, CmR, oriT plasmid carrying TLCФ & $\begin{array}{c}\text { (Midonet et } \\
\text { al., } \\
2014) \\
\end{array}$ \\
\hline $\begin{array}{c}\text { pFB0005 (pET- } \\
\text { Gate2) }\end{array}$ & $\begin{array}{l}\text { Backbone for Golden gate assembly for gene targeting in Bacillus } \\
\text { subtilis }\end{array}$ & $(51)$ \\
\hline pSM11 & Derivative of pTLC8 in which $a_{t t} p_{T L C}$ was replaced by Bsa1-ccdb-Bsa1 & This study \\
\hline pSM12 & Derivative of pTLC10 lacking all TLCФ nucleotides from 325 to 4137 & This study \\
\hline pSM15 & $\begin{array}{l}\text { Derivative of pSM12 in which attp } \text { TLC }_{\text {Bas }} \text { waslaced by Bsa1-ccdb- } \\
\text { Bsa1 }\end{array}$ & This study \\
\hline pSM17 & Derivative of pSM11 carrying dif1 & This study \\
\hline pSM18 & Derivative of pSM15 carrying dif1 & This study \\
\hline pCM153 & $\begin{array}{l}\text { pBR322 + MBP-6His-TEVsite-VC1465 under T7 promotor and lacO } \\
\text { operator + KanR }\end{array}$ & (23) \\
\hline pCM157 & $\begin{array}{l}\text { pBR322 + MBP-6His-TEVsite-V. cholerae XerC under T7 promoter } \\
\text { and lacO operator }+ \text { KanR }\end{array}$ & (23) \\
\hline pCM162 & $\begin{array}{l}\text { pBR322 + MBP-6His-TEVsite-V. cholerae XerD under T7 promoter } \\
\text { and lacO operator + KanR }\end{array}$ & (23) \\
\hline pCM163 & $\begin{array}{l}\text { pBR322 + MBP-6His -TEVsite-V. cholerae XerC catalytic mutant (KQ } \\
\text { mutation) under T7 promoter and lacO operator + KanR }\end{array}$ & (23) \\
\hline pCM164 & $\begin{array}{l}\text { pBR322 + MBP-6His -TEVsite-V. cholerae XerD catalytic mutant (KQ } \\
\text { mutation) under T7 promoter and lacO operator + KanR }\end{array}$ & (23) \\
\hline
\end{tabular}

Table S4. Oligonucleotide list.

\begin{tabular}{|c|c|c|}
\hline \multicolumn{2}{|c|}{ Cloning } & \multirow{2}{*}{\begin{tabular}{|l|} 
Template \\
pTLC8
\end{tabular}} \\
\hline 2572 & AAGACTACGAAACACAAACC & \\
\hline 2321 & AGGATCCCCCGGGCTGCAGGAATTCG & pTLC8 \\
\hline 4059 & ATATCGAATTCCTGCAGCCCGGGGGATCCTgagaccttccggctcgtatg & pFB0005 \\
\hline 4060 & GGTTAAGCTTGGTTTGTGTTTCGTAGTCTTgagacccgggagcagacaag & pFB0005 \\
\hline \multicolumn{3}{|c|}{ Library construction } \\
\hline 4066 & $\begin{array}{l}\text { gttacaggtctcaatcctagtgcgcattatgtatgnnnnnnnngtgaagactgagacccaatgaga } \\
\text { gcgtgcgaataaggatggatataccgaca }\end{array}$ & Fill in reaction with 4069 \\
\hline 4067 & $\begin{array}{l}\text { gttacaggtctcaatcctagtgcgcattatgtatgtagnnnnnnnnaagactgagacccaatgaga } \\
\text { gcgtgcgaataaggatggatataccgaca }\end{array}$ & Fill in reaction with 4069 \\
\hline 4068 & $\begin{array}{l}\text { gttacaggtctcaatcctagtgcgcattatgtatgnnnnnaannngaagactgagacccaatgaga } \\
\text { gcgtgcgaataaggatggatataccgaca }\end{array}$ & Fill in reaction with 4069 \\
\hline 4177 & \begin{tabular}{|l|} 
gttacaggtctcaatcctagtgcgcattatgtatgttatgttaaataagactgagacccaatgagagcg \\
tgcgaataaggatggatataccgaca
\end{tabular} & Fill in reaction with 4069 \\
\hline 4069 & tgtcggtatatccatccttattcgcacgctc & Reverse for fill in \\
\hline \multicolumn{3}{|c|}{ Sequencing primers } \\
\hline 4103 & $\begin{array}{l}\text { aatgatacggcgaccaccgagatctacactctttccctacacgacgctcttccgatctcttggtttgtgt } \\
\text { ttcgtagtct }\end{array}$ & P5, Plasmid/Integrated \\
\hline 4104 & $\begin{array}{l}\text { aatgatacggcgaccaccgagatctacactctttccctacacgacgctcttccgatctccttggtttgtg } \\
\text { tttcgtagtct }\end{array}$ & P5, Plasmid/Integrated \\
\hline
\end{tabular}




\begin{tabular}{|c|c|c|}
\hline 4105 & $\begin{array}{l}\text { aatgatacggcgaccaccgagatctacactctttccctacacgacgctcttccgatctatcttggtttgt } \\
\text { gtttcgtagtct }\end{array}$ & P5, Plasmid/Integrated \\
\hline 4106 & $\begin{array}{l}\text { aatgatacggcgaccaccgagatctacactctttccctacacgacgctcttccgatcttgtccttggttt } \\
\text { gtgtttcgtagtct }\end{array}$ & P5, Plasmid/Integrated \\
\hline 4174 & $\begin{array}{l}\text { caagcagaagacggcatacgagatatcagtgtgactggagttcagacgtgtgctcttccgatccggt } \\
\text { atcgataagcttgatatcg }\end{array}$ & P7, Plasmid \\
\hline 4175 & $\begin{array}{l}\text { caagcagaagacggcatacgagatcgcctggtgactggagttcagacgtgtgctcttccgatccggt } \\
\text { atcgataagcttgatatcg }\end{array}$ & P7, Plasmid \\
\hline 4176 & $\begin{array}{l}\text { caagcagaagacggcatacgagatattccggtgactggagttcagacgtgtgctcttccgatccggta } \\
\text { tcgataagcttgatatcg }\end{array}$ & P7, Plasmid \\
\hline 4243 & $\begin{array}{l}\text { caagcagaagacggcatacgagataggaatgtgactggagttcagacgtgtgctcttccgatccggt } \\
\text { atcgataagcttgatatcg }\end{array}$ & P7, Plasmid \\
\hline 4244 & $\begin{array}{l}\text { caagcagaagacggcatacgagatagctaggtgactggagttcagacgtgtgctcttccgatccggt } \\
\text { atcgataagcttgatatcg }\end{array}$ & P7, Plasmid \\
\hline 4245 & $\begin{array}{l}\text { caagcagaagacggcatacgagatattatagtgactggagttcagacgtgtgctcttccgatccggta } \\
\text { tcgataagcttgatatcg }\end{array}$ & P7, Plasmid \\
\hline 4178 & $\begin{array}{l}\text { caagcagaagacggcatacgagatagctaggtgactggagttcagacgtgtgctcttccgatcttatg } \\
\text { gcagggtgaaacgcagg }\end{array}$ & P7, Integrated \\
\hline 4179 & $\begin{array}{l}\text { caagcagaagacggcatacgagatcgattagtgactggagttcagacgtgtgctcttccgatcttatg } \\
\text { gcagggtgaaacgcagg }\end{array}$ & P7, Integrated \\
\hline 4180 & $\begin{array}{l}\text { caagcagaagacggcatacgagatgctcatgtgactggagttcagacgtgtgctcttccgatcttatg } \\
\text { gcagggtgaaacgcagg }\end{array}$ & P7, Integrated \\
\hline 4246 & $\begin{array}{l}\text { caagcagaagacggcatacgagattgttgggtgactggagttcagacgtgtgctcttccgatcttatg } \\
\text { gcagggtgaaacgcagg }\end{array}$ & P7, Integrated \\
\hline 4247 & $\begin{array}{l}\text { caagcagaagacggcatacgagatgccatggtgactggagttcagacgtgtgctcttccgatcttatg } \\
\text { gcagggtgaaacgcagg }\end{array}$ & P7, Integrated \\
\hline 4248 & $\begin{array}{l}\text { caagcagaagacggcatacgagatatcgtggtgactggagttcagacgtgtgctcttccgatcttatg } \\
\text { gcagggtgaaacgcagg }\end{array}$ & P7, Integrated \\
\hline 4259 & $\begin{array}{l}\text { gttacaggtctcaatcctngtgcgcattatgtatgtagagaaagtgaagactgagacccaatgagag } \\
\text { cgtgcgaataaggatggatataccgaca }\end{array}$ & P7, Integrated \\
\hline 4260 & $\begin{array}{l}\text { gttacaggtctcaatcctantcgcattatgtatgtagagaaagtgaagactgagacccaatgagagc } \\
\text { gtgcgaataaggatggatataccgaca }\end{array}$ & P7, Integrated \\
\hline 4261 & $\begin{array}{l}\text { gttacaggtctcaatcctagngcgcattatgtatgtagagaaagtgaagactgagacccaatgagag } \\
\text { cgtgcgaataaggatggatataccgaca }\end{array}$ & P7, Integrated \\
\hline 4262 & $\begin{array}{l}\text { gttacaggtctcaatcctagtncgcattatgtatgtagagaaagtgaagactgagacccaatgagag } \\
\text { cgtgcgaataaggatggatataccgaca }\end{array}$ & P7, Integrated \\
\hline 4263 & $\begin{array}{l}\text { gttacaggtctcaatcctagtgngcattatgtatgtagagaaagtgaagactgagacccaatgagag } \\
\text { cgtgcgaataaggatggatataccgaca }\end{array}$ & P7, Integrated \\
\hline 4264 & $\begin{array}{l}\text { gttacaggtctcaatcctagtgcncattatgtatgtagagaaagtgaagactgagacccaatgagag } \\
\text { cgtgcgaataaggatggatataccgaca }\end{array}$ & P7, Integrated \\
\hline 4265 & $\begin{array}{l}\text { gttacaggtctcaatcctagtgcgnattatgtatgtagagaaagtgaagactgagacccaatgagag } \\
\text { cgtgcgaataaggatggatataccgaca }\end{array}$ & P7, Integrated \\
\hline 4266 & $\begin{array}{l}\text { gttacaggtctcaatcctagtgcgcnttatgtatgtagagaaagtgaagactgagacccaatgagag } \\
\text { cgtgcgaataaggatggatataccgaca }\end{array}$ & P7, Integrated \\
\hline 4267 & $\begin{array}{l}\text { gttacaggtctcaatcctagtgcgcantatgtatgtagagaaagtgaagactgagacccaatgagag } \\
\text { cgtgcgaataaggatggatataccgaca }\end{array}$ & P7, Integrated \\
\hline 4268 & $\begin{array}{l}\text { gttacaggtctcaatcctagtgcgcatnatgtatgtagagaaagtgaagactgagacccaatgagag } \\
\text { cgtgcgaataaggatggatataccgaca }\end{array}$ & P7, Integrated \\
\hline 4269 & $\begin{array}{l}\text { gttacaggtctcaatcctagtgcgcattntgtatgtagagaaagtgaagactgagacccaatgagag } \\
\text { cgtgcgaataaggatggatataccgaca }\end{array}$ & P7, Integrated \\
\hline \multicolumn{3}{|c|}{ In vitro recombination } \\
\hline 3418 & & Annealing with 3419 \\
\hline 3419 & & Annealing with 3418 \\
\hline 2206 & GCTTGATATCGAATTCCTGCAGCCC & pSM17 \\
\hline
\end{tabular}


bioRxiv preprint doi: https://doi.org/10.1101/2021.09.09.459576; this version posted September 9, 2021. The copyright holder for this preprint (which was not certified by peer review) is the author/funder, who has granted bioRxiv a license to display the preprint in perpetuity. It is made available under aCC-BY-NC-ND 4.0 International license.

\begin{tabular}{|l|l|l|}
\hline $\mathbf{2 4 8 1}$ & CCCGAGCTCCCTGCCAATCCTTACGATG & pSM17 \\
\hline $\mathbf{2 4 7 9}$ & CCCGAGCTCTCAAAAGCTCAGCCTCCTAC & pTLC8 \\
\hline $\mathbf{1 1 1 0}$ & CTAGACAGCGCTTTTCCGCTGCATAAC & pTLC8 \\
\hline
\end{tabular}

